Check for updates

Cite this: RSC Adv., 2018, 8, 37915

\title{
Recent developments in polymeric electrospun nanofibrous membranes for seawater desalination
}

\author{
Mantsopa Koena Selatile, ${ }^{\text {ab }}$ Suprakas Sinha Ray, (D)*ac Vincent Ojijo ${ }^{a}$ \\ and Rotimi Sadiku
}

Seawater desalination is a promising strategy that offers an abundant and reliable source of clean fresh water. Nanotechnology, in terms of nanoparticles or electrospun nanofibrous membranes, for watertreatment or desalination applications, is a new concept that has rapidly grown in interest as a method for improving performance by enhancing the surface properties of membranes. Here, we report a critical review on recent developments in membrane-fabrication methods for seawater desalination technologies, focusing mainly on the electrospinning technique. High-performance membranes that address ongoing permeability concerns, while maintaining membrane selectivity, need further study and development. Considering that the world today is faced with energy-shortage crises, these membranes also need to be energy efficient. As electrospinning is considered to be a feasible method for the production of desalination membranes, this technique requires appropriate optimization and the structural properties of the membranes produced need to be controlled in order to tailor their properties to those desired for well-known desalination technologies, such as reverse osmosis and membrane distillation. Moreover, there is a need to understand the influence of membrane structure on performance, and the latest trends in their use as high-performance desalination membranes.

Received 7th September 2018
Accepted 5th November 2018

DOI: $10.1039 / \mathrm{c} 8 \mathrm{ra07489e}$

rsc.li/rsc-advances

\section{Introduction}

Clean water is a vital resource for human consumption and agriculture, as well as feed water for industrial processes, which are the most common daily activities that exploit what is arguably the most valuable global resource. However, due to factors such as global warming (since climate change affects the hydrologic cycle), industrialization, and freshwater contamination, among others, existing water sources such as surface water (rivers and dams), recycled wastewater, and some underground sources, are also becoming depleted owing to rising demand and decreasing rainfall for freshwater supply. Wastewater recycling is also unable to meet demand, depending on the extent or severity of contamination. Although several measures that relieve water-supply stresses exist, such as water conservation, infrastructure repair, and improved catchment and distribution systems, they need to be augmented accordingly. The water crisis facing the world today, which affects economic growth and human health, has led to alternative means for obtaining fresh water. The oceans are the Earth's largest available source of water and account for $97 \%$ of the

${ }^{a}$ DST-CSIR National Centre for Nanostructured Materials, Council for Scientific and Industrial Research, Pretoria 0001, South Africa. E-mail: rsuprakas@csir.co.za; ssinharay@uj.ac.za

${ }^{b}$ Division of Polymer Technology, Department of Chemical, Metallurgical and Materials Engineering, Tshwane University of Technology, South Africa

'Department of Applied Chemistry, University of Johannesburg, Doornfontein 2028, Johannesburg, South Africa available water; they represent a more reliable source of water because they are unaffected by changing rainfall patterns. The remaining $3 \%$ is freshwater, of which two thirds is frozen in glaciers and polar ice caps, and the rest is found in underground water reservoirs. ${ }^{1,2}$ With this in mind, seawater treatment represents a more-reliable approach that has catalyzed research into alternative technologies; as a result, seawater desalination is a popular field of interest. Desalination can be performed on inland underground brackish water or seawater, of which brackish-water treatment is less feasible than seawater treatment. Seawater desalination offers an abundant, steady supply of highquality water. The desalination of seawater is commonly used in arid regions and deserts, but due to worldwide water stresses has become more popular in developing counties in recent years. ${ }^{3}$ Desalination involves the evaporation of seawater, thereby removing salts, minerals, and other impurities, to produce fresh water for domestic and industrial use. ${ }^{4}$

The high salt content of seawater makes it unfit for consumption and, at the same time, makes desalination a costly process. The salinity or salt content of seawater is due to the presence of about $3.5 \% \mathrm{NaCl}$, which is equivalent to $35 \mathrm{~g} \mathrm{~L}^{-1}$ or $35000 \mathrm{ppm}$. Drinking water should have a $\mathrm{NaCl}$ concentration of less than $280 \mathrm{ppm}^{3,5}$ Seawater-desalination methods are classified into thermal, mechanical, electrical, and chemicalenergy processes. Desalination technologies are either thermally or pressure driven and include a variety of technologies, such as multi-stage flash (MSF) distillation, multi-effect 
distillation (MED), mechanical vapor-pressure-compression (MVC) distillation, membrane distillation (MD), reverse osmosis (RO), electrodialysis (ED), and nanofiltration (NF) processes. Thermally based techniques are first-generation technologies, while membrane-based methods are secondgeneration. Membrane-based methods make use of nanotechnology and membrane science, are more suitable for desalination, and are more economical due to lower energy requirements. These methods include microfiltration, (MF), ultrafiltration (UF), engineered osmosis (EO) (reverse and forward osmosis), and membrane distillation (MD). Reverse osmosis (RO) is the most preferred pressure-driven process, while membrane distillation (MD) is the most preferred thermally driven process. Therefore both RO and MD methods have been intensively researched due to their high salt-removal efficiencies (i.e., $>98 \% \mathrm{NaCl}$ removal) and, as a consequence, are the most used seawater-desalination techniques for the production of drinking water. Other methods, such as nanofiltration, are mainly employed for lower salt-content removal $\left(20-80 \% \mathrm{NaCl}\right.$ removal) and mostly for brackish water. ${ }^{6-9}$ Desalination has become more practical and economical, and research into membrane-based desalination methods has gradually grown over the past ten years (Fig. 1).

The process performance of membrane-based desalination methods, in terms of permeability (water flux) and separation (selectivity or salt rejection), depends highly on the characteristics of the membrane. With continuous improvements achieved through the optimization of membrane characteristics, membrane processes are becoming more performance efficient (e.g., improved permeability) and, as a consequence, use less energy than thermally driven processes.

Nanofibrous membranes produced by electrospinning (ENMs) form part of the nanotechnology-based methods used to fabricate novel separation membranes. ENMs possess

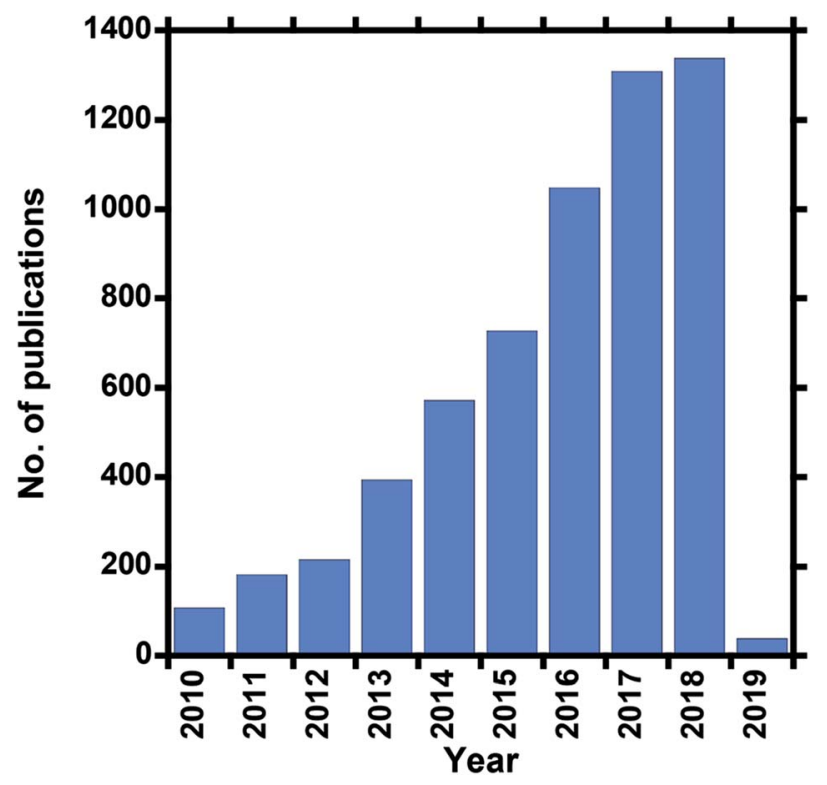

Fig. 1 Number of publications over the past ten years that involve electrospun desalination membranes (Scopus search, 05 October 2018). exceptional features because they can be used to produce multifunctional water-purification materials. However, for desalination purposes, their highly porous structures with broad pore-size distributions (micron-sized pores) limit their ability to reject dissolved salts, such as $\mathrm{NaCl}$, from water. They are therefore limited to MF-separation applications involving large/microsized particles, or as pre-filters prior to delicate RO processes. Through modification, the water-treatment applications of these membranes can be further extended to the removal of morecomplex colloidal solutions, such as oil/water suspensions, which involves the rejection of an organic solute. Modification of an ENM surface with a thin selective coating layer through crosslinking results in the formation of a composite membrane with smaller pores that can be used for UF separations. Further modification through interfacial-polymerization coating leads to nonporous composite membranes that can be used in NF and RO applications. Interfacial polymerization produces a polyamide coating as an ultrathin top selective layer on the ENM surface, resulting in a thin-film nanofibrous composite (TFNC) membrane; such a membrane can be used in RO and MD separations, which are two commonly used desalination methods. The coated layer of a TFNC membrane can be further modified to improve permeability and salt rejection (permselectivity) through the inclusion of nanoparticles (NPs). The incorporation of NPs into ENMs help to enhance performance (both flux and salt rejection); therefore, the use of nanotechnology in existing desalination technologies can result in high membrane performance. This review article examines the development of polymeric ENMs for seawater desalination processes, particularly RO and MD, which are the most commonly used and most efficient methods. The influences of membrane structure on performance, and the latest trends in their use as high-performance desalination membranes, are also discussed.

\section{Water treatment by membrane- based technologies and materials used}

Membrane separation is a technology that selectively separates materials using the pores of a membrane that acts as a physical barrier. The semi-permeable membrane allows water to pass through the membrane while retaining solute particles through sieving and diffusion mechanisms. The pressure applied on the feed side of the membrane serves as the driving force that separates water into two streams, namely the permeate (purified water) and the retentate (rejected concentrated salt solution), which is brine in a desalination process. Membrane technologies are classified according to separation principles or driving force, and membrane properties (e.g., pore size, permeability, and selectivity). Pressure-driven membrane-based processes are classified according to membrane pore size and their corresponding solute particle sizes, namely: microfiltration (MF) > ultrafiltration (UF) > nanofiltration (NF) > reverse osmosis (RO), with the larger pore/particle sizes used for MF and the smallest for RO (Fig. 2). The required applied pressure (trans-membrane pressure) differs according to the filtration process, and 
increases with decreasing membrane-pore size. Consequently, RO operates at the highest hydrostatic pressure. ${ }^{\mathbf{1 0 , 1 1}}$

\subsection{Pressure-driven processes and the role of the ENM}

2.1.1. MF membranes. Microfiltration membranes, as the name implies, have pores that range in size from sub-micron to micron (100 nm to $10 \mu \mathrm{m})$, and fibers size in the $100 \mathrm{~nm}$ to $1 \mu \mathrm{m}$ range, and are capable of capturing large particles $(>\sim 1 \mu \mathrm{m})$ through the sieving mechanism. These membranes are usually used as pre-filters for UF processes. ENMs are good MFmembrane pre-filters as they provide high internal surface areas that result in high solute-holding capacities and high average pore sizes that can be reduced with increasing thickness. As they operate as single layers, they are required to be mechanically stable. In fact, in an early study, an electrospun PVDF membrane was used as a liquid-filtration MF for the removal of 1-10 $\mu \mathrm{m}$ microparticles from a water stream. However, fouling caused by irreversible cake filtration was problematic. Irrespective of surface filtration or deep filtration, the rejected particles remained attached to the surface resulting in pore blockage; as a result these membranes were unable to be reused..$^{10,12,13}$

2.1.2. UF membranes. UF membranes have pore sizes in the $10-100 \mathrm{~nm}$ range, therefore water is forced to pass through their nanopores under hydrostatic pressure, with only lowmolecular-weight particles and dissolved ions passing through. UF membranes are mainly use for the separation of small colloids, such as oil/water microemulsions, bacteria, and viruses, in the paint and food industries. Like MF membranes, these UF membranes are also used as pre-treatment membranes for NF- and RO-desalination processes, as the RO process requires high-quality feed water for effective separation. Fig. 3(a) shows a typical UF phase-inversion membrane with dead-end micropores. ENMs on the other hand, consist of interconnected pore structures that serve as pathways for water that freely flows through the membrane; therefore, they have lower hydraulic resistances than phase-inversion membranes. Ideal UF membranes exhibit narrow pore-size distributions in addition to high porosities. ${ }^{\mathbf{1 4 1 5}}$ The use of an ENM in place of a phase-inversion membrane during UF alleviates the low flux due to low porosity, the inefficient removal of particles from the water stream (rejection), and the high fouling rate due to the broad pore-size distribution experienced by a conventional UF membrane produced by phase inversion. A thin-film coating can be applied to mitigate the broad pore-size distribution, as shown in Fig. 3(b); however, ENMs also have broadly distributed pore sizes and suffer high levels of fouling as a consequence. To overcome the low porosities of these phase-inversion membranes and to increase flux, a thin film layer is coated onto an electrospun, porous, hydrophilic support layer that replaces the conventional membrane, as shown in Fig. 3(c). The porosities of ENMs help to enhance the effective flow path

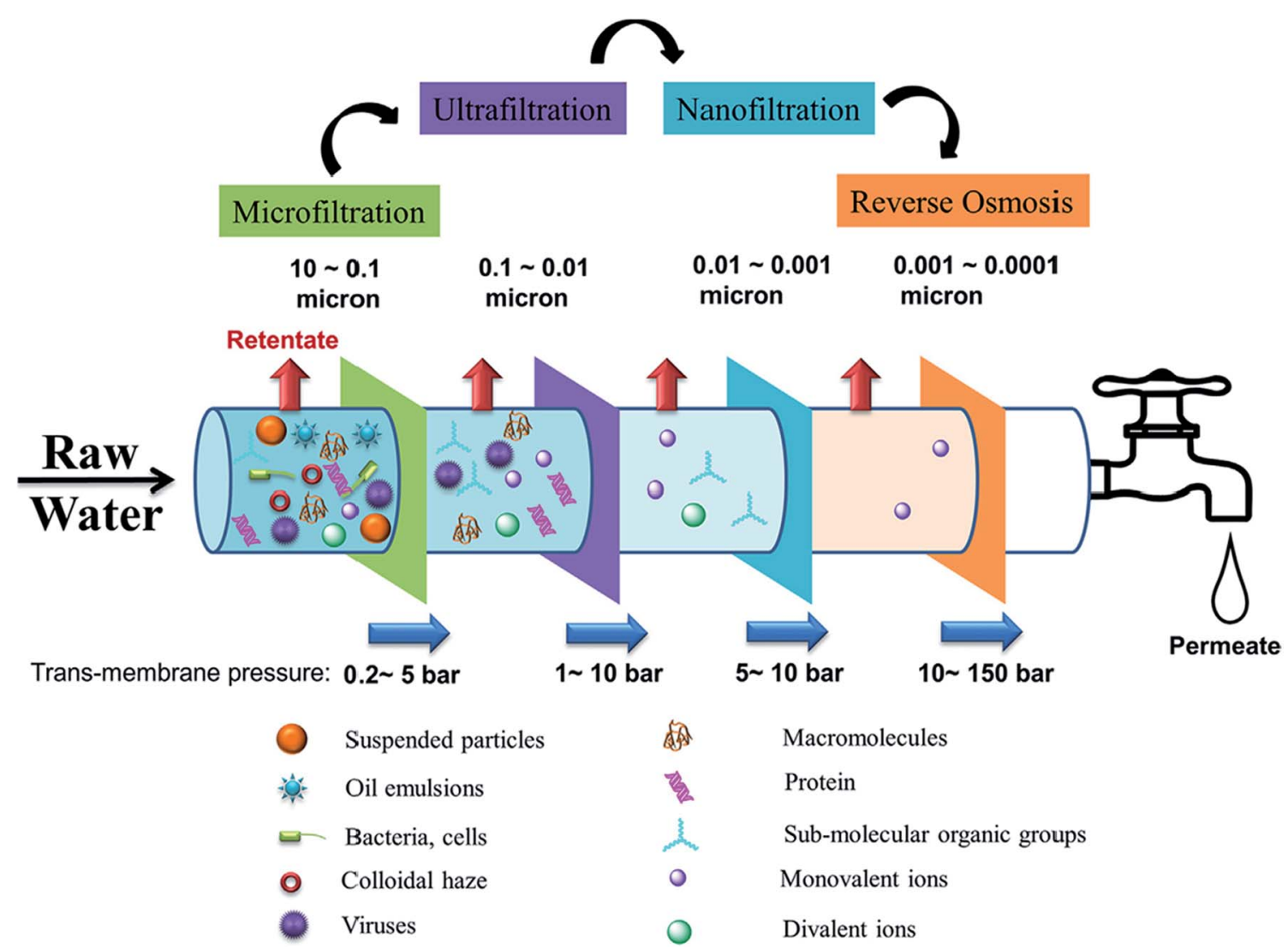

Fig. 2 Pressure-driven membrane processes for water treatment technologies, showing the particles effectively captured by each process along with the pore sizes of the membranes used for each process. Reproduced with permission from ref. 10. Copyright 2018, Elsevier Science Ltd. 

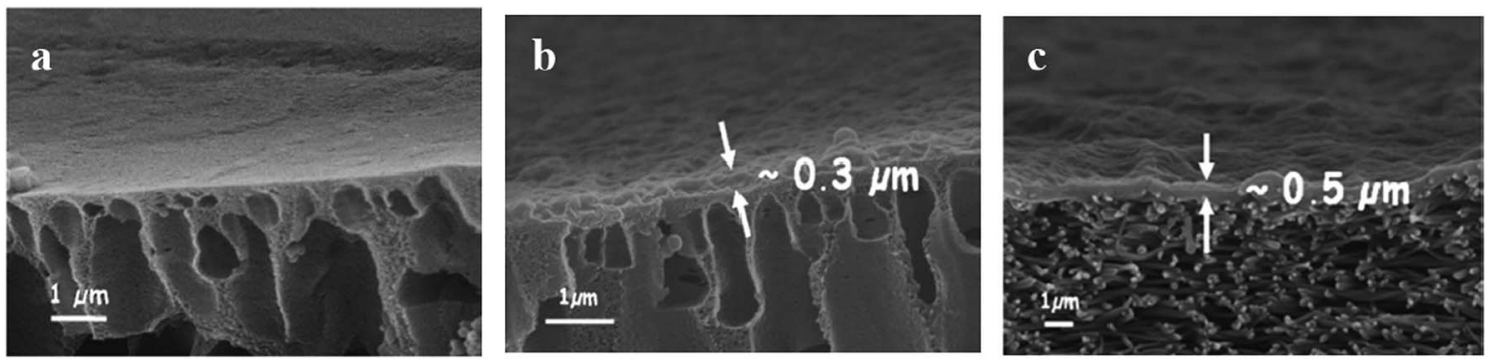

Fig. 3 Cross-sections of (a) the mid layer of a TFC UF membrane prepared by the phase-inversion method, (b) a phase-inversion mid layer coated with a thin selective layer (cross-linked or grafted), and (c) an ENM mid layer coated with a thin film (TFNC). Reproduced with permission from ref. 17. Copyright 2006, Elsevier Science Ltd.

through the skin layer. Replacement of a conventional phaseinversion membrane by an ENM in a typical three-layer membrane system results in a composite membrane (i.e., a nanofibrous composite UF membrane or TFNC membrane). The layers consist of a thin film, which is a porous substrate on a support, namely a crosslinked or a grafted top layer (using a highly water-permeable/hydrophilic polymer, such as chitosan or a PVA hydrogel), an ENM mid-layer (e.g., hydrophobic PVDF or polyether sulfone (PES)), and a commercial nonwoven microfiber substrate. ${ }^{16}$ These composite membranes provide fluxes as high as $30 \mathrm{Lm}^{-2} \mathrm{~h}^{-1}$ and rejections of $99 \%$. Both hydrophobic and hydrophilic polymers have been used as electrospun porous supports or mid layers during UFmembrane fabrication; however hydrophilic substrates are preferred due to their superior antifouling properties. Membrane surfaces have also been modified to enhance water permeability, hydrophilicity, and antifouling properties. ${ }^{10}$

2.1.3. NF membranes. NF membranes have pore sizes in the $1-10 \mathrm{~nm}$ range (for $0.1-1 \mathrm{~nm}$-sized particles). ${ }^{18,19}$ Separation is driven by a high trans-membrane pressure facilitated by sieving or steric hindrance, and electrostatic effects. ${ }^{10}$ These membranes are proficient for the removal of organic contaminants and large divalent ions from water, while smaller monovalent salts, such as $\mathrm{NaCl}$, pass through; consequently, taste, odor, and color are removed. NF membranes are constructed as three-layer composite membranes, but the thin film is produced by interfacial polymerization (IP) in which a top ultrathin dense layer is deposited onto a more-porous ENM that replaces the phase-inversion membrane and is supported by a nonwoven commercial substrate; a TFNC is formed in this manner (Fig. 4). The large pore size, and the high surface and internal porosities of an ENM, as a mid-layer, enhance the permeability of the TFNC membrane. In addition, flux increases with increasing fiber diameter, albeit at the cost of salt rejection. Nanofiltration has proven its potential for the desalination of water, but only in water of lower salt content, such as brackish water. This process has become more popular than RO due to its lower pressure requirements, and hence, low energy requirements, while maintaining high fluxes. ${ }^{10}$

2.1.4. Reverse osmosis (RO). During reverse osmosis (membrane pore sizes of $0.1-1 \mathrm{~nm}$ ), the feed is transported against the natural flow in a concentrated solution; the water therefore flows from the concentrated salt water (feed solution) to the dilute water (draw solution). RO has been the most popular method for the production of fresh water by seawater desalination since the 1950s. The required hydraulic pressure of about 0.7-5.5 MPa is sufficient to overcome the osmotic pressure of the salt solution through the membrane. The use of improved high-permeability membranes has advantages that include efficiency and reduced power consumption. TFC polyamide RO membranes, with good fluxes and salt-rejection properties, are typical membranes used in desalination applications. Unlike NF membranes that mainly retain divalent ions and multivalent salts such as sodium sulfate and calcium sulfate, RO membranes are NF membranes that are capable of rejecting monovalent salts, such as sodium chloride ( $\mathrm{NaCl})$; hence RO is the most effective pressure-driven desalination method. ${ }^{11}$ The disadvantages of RO include scaling, brine disposal, and boron removal.

\subsection{Thermally driven membrane processes}

Thermally driven membrane processes are evaporative desalination techniques in which energy is used to heat water, which then evaporates and condenses into clean purified water. As this is a rather energy-intensive process, it is more viable in refineries or power plants where waste heat is used as the heat source. Nevertheless, membrane distillation (MD) operates at a lower temperature than a typical thermal-desalination process, and at a lower hydrostatic pressure than RO. As the name implies, it combined membrane-based and thermally based desalination processes. MD is a thermally driven nonisothermal process that makes use of a microporous hydrophobic membrane as a barrier (for water as the liquid phase),

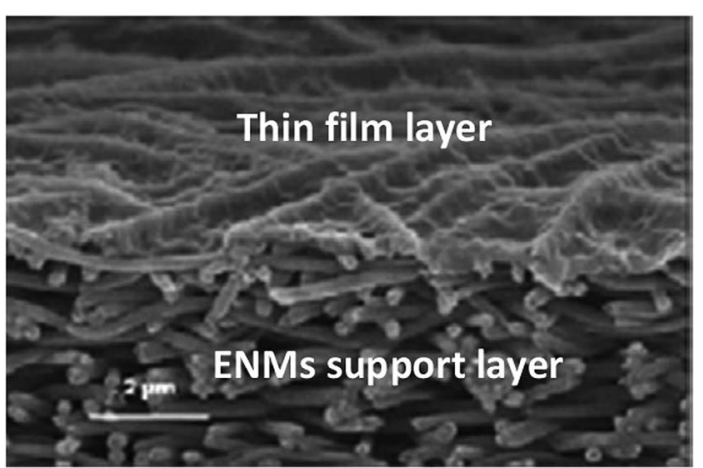

Fig. 4 A NF TFNC membrane. Reproduced with permission from ref. 10. Copyright 2018, Elsevier Science Ltd. 
and only allows the water-vapor phase to pass through. Vapor molecules permeate through the membrane from the warmer high-vapor-pressure side to the cooler low-vapor-pressure side. It is an MF process with membrane pore sizes in the microfiltration range $(0.1-1 \mu \mathrm{m})$ and is driven by the partial vaporpressure difference across a microporous hydrophobic membrane (which is induced by the temperature difference between the feed side and the permeate side) produced by phase inversion (PVDF, PP, and PTFE capillary/flat-sheet types), which are now as conventional as ENMs. ${ }^{20,21}$

Membrane distillation is a promising desalination technology that was developed following the evolution of RO. It is also regarded to be a third-generation desalination technique. ${ }^{22}$ MD addresses some of the limitations of RO, such as brine concentration, in order to maximize water recovery (salt rejection) at high fluxes. The high rejection of non-volatile components and the use of lower energy than RO and conventional distillation processes make $\mathrm{MD}$ an attractive separation method, as it offers more advantages over other desalination technologies. MD does not require any transmembrane hydrostatic pressure and therefore self-sustained or single-layer ENMs that do not require thin-film coatings can be used. However, it is worth noting that hydraulic pressure is required, but at levels far less than those used in pressure-driven processes, such as RO. ${ }^{5} \mathrm{MD}$ is widely used for industrial water desalination in a variety of industries, including brackish and seawater desalination, RO-brine treatment, resource recovery, wastewater reuse, and the treatment of mine water and radioactive waste, among others.

MD can be configured in four ways: direct-contact membrane distillation (DCMD), air-gap membrane distillation (AGMD), sweeping-gas membrane distillation (SGMD), and vacuum membrane distillation (VMD), which are determined by the manner in which the vapor-pressure gradient across the membrane arises, as summarized in Fig. 5.,.$^{3,1023}$

Direct-contact membrane distillation (DCMD) is the most popular due to its simple set-up and fabrication process; the feed directly contacts the hot side of the membrane surface. Volatile water molecules evaporate at the liquid/vapor interface at the high-temperature-feed side, then cross through the hydrophobic porous membrane, and finally condense at the cold permeate-side of the membrane. This process requires low operating temperatures as the water boils at $30-80{ }^{\circ} \mathrm{C}$, which is below its boiling point at atmospheric pressure. ${ }^{\mathbf{1 0 2 4}}$ The feed is only in direct contact with the hot side of the membrane surface in air-gap membrane distillation (AGMD), due to stationary air present between the membrane and the condensation surface. This method is operated using a membrane and an air gap through which the water vapor permeates. The warm water feed and the cooling water create a temperature difference between the two sections. A typical example is the 25 to $83{ }^{\circ} \mathrm{C}$ temperature gradient used during AGMD with an electrospun PVDF. Sweeping-gas membrane distillation (SGMD) uses an inert gas to sweep the vapor at the permeate side of the membrane such that it condenses on the outside of the membrane module. A moving gas barrier, as in AGMD, is used to reduce heat loss and to enhance the mass- transfer coefficient. Vacuum membrane distillation (VMD) uses a pump to create a vacuum on the permeate side of the membrane. ${ }^{3,8}$

2.2.1. Advantages of MD. It uses less energy than other conventional distillation processes, and the hydrostatic pressure required is less than that used in pressure-driven processes such as RO. With a $100 \%$ theoretical separation rate for nonvolatiles such as $\mathrm{NaCl}$ (salt rejection), $\mathrm{MD}$ is expected to produce high-quality water. Lower feed velocities result in lower energy costs, as low-grade heat can be used to drive the process. $\mathrm{MD}$ is less prone to fouling due to the hydrophobic nature of the membranes used and their large pore sizes compared to RO and NF. MD can be assimilated with other processes such as RO and NF to overcome their limitations; hence it can also be used as an additional process that increases water recovery from the ROwaste or concentrated-brine stream. ${ }^{5}$

2.2.2. Disadvantages of MD. Owing to their low permeation fluxes compared RO-process membranes, there is a need to further develop and improve MD membranes. Prolonged use results in membrane deterioration due to concentration and temperature polarization. There is also a high chance of pore wetting during operation, and increases in mass-transfer resistance due to trapped air within the membrane, which also limits the flux of the MD permeate. MD is also associated with high heat loss through the membrane by conduction. ${ }^{3}$

\section{Membrane fabrication by electrospinning}

The membrane-fabrication technique is highly important as it directly affects the properties of the membrane as well as its water-desalination performance. Amongst popular techniques used to fabricate polymeric desalination membranes (phase inversion, interfacial polymerization, stretching, and track etching), electrospinning offers a number of advantages, including control over nanofiber morphology, which leads to membranes with the desired properties. Some of the unique characteristics of electrospun membranes include nanodimensional fibers, high specific surface areas, high porosities, and highly oriented or aligned nanofibers, which benefit the nanofiber-based construction significantly. The surface and structural properties of a membrane, such as its thickness, porosity, pore-size distribution, polymer crystallinity, and hydrophobicity/hydrophilicity, are all affected by fiber diameter and morphology, which can also be controlled by the electrospinning parameters, namely polymer-solution concentration or viscosity, conductivity, voltage, and flow rate. ${ }^{\mathbf{1 2 , 2 5}}$

Electrospinning involves uniaxial stretching of viscoelastic polymer solution by repulsive electrostatic forces under electric field, and is fundamentally governed by a number of operating conditions and solution properties. ${ }^{26}$ It all starts with a high molecular weight polymer that dissolves in a suitable solvent to form a homogeneous solution with sufficient chain entanglements. The solution properties are dependent on the solvent and polymer itself and the selection of the operating conditions for a sufficiently high polymer chain entanglement solution, 


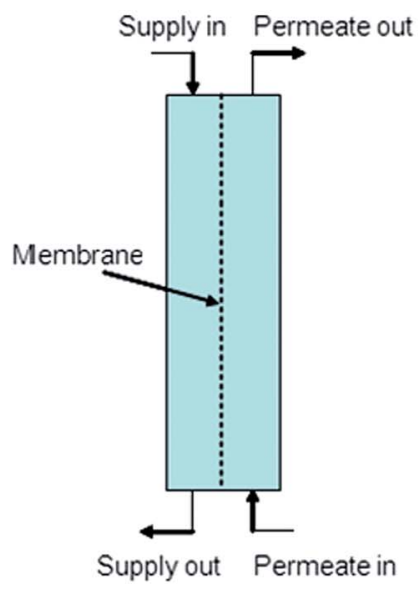

DCMD configuration

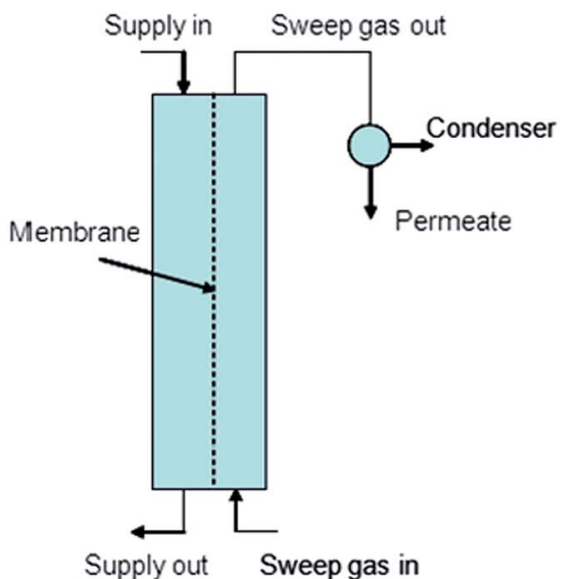

SGMD configuration

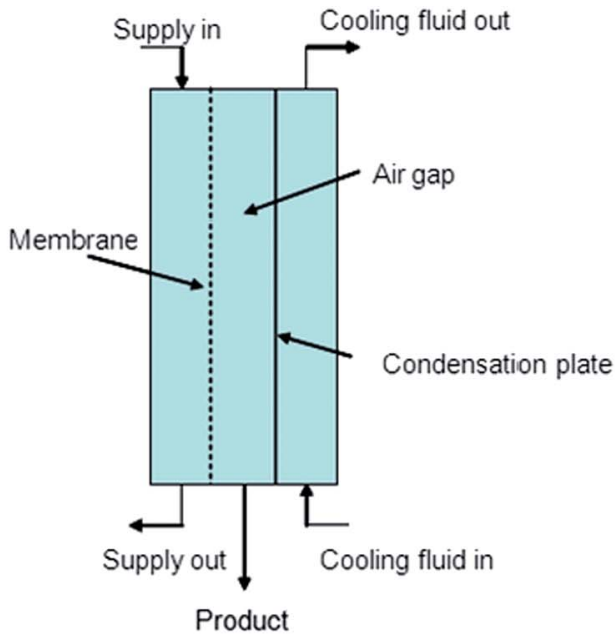

AGMD configuration

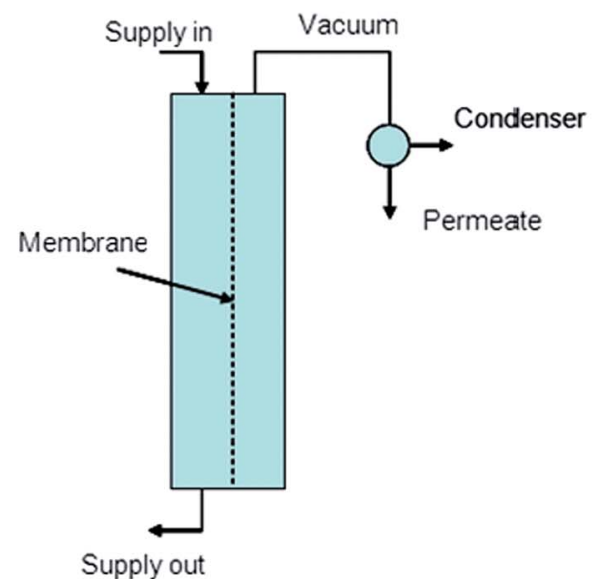

VMD configuration

Fig. 5 Depicting various MD configurations. Reproduced with permission from ref. 23. Copyright 2006, Elsevier Science Ltd.

through parameter optimization results in the production of continuous network of fibers. One of the advantages of electrospinning is that it allows for easy tuning of parameters in order to obtain the fibres with desired physical properties and performance. ${ }^{27}$

Electrospinning consists of three basic components, namely: high voltage supply, syringe connected to a needle and a metal collector (Fig. 6). The syringe, containing the polymer solution with a blunt needle tip (spinneret) is connected as a positive electrode. This passes charges to the polymer solution droplet suspended at the tip. The collector offers a platform for deposition of fine micro/nanosized fibers and can either be grounded or carry a charge opposite to the spinneret, most often, negative charge. At the initial stages of the process, the polymer solution in the syringe is pushed to the needle tip and remains suspended. Surface tension keeps the round hemispherical droplet attached to the needle tip. Once the voltage is increased, the hemispherical droplet elongates into a conical shape and forms a Taylor cone, as soon as the voltage reaches a critical value. As the high voltage source creates an electrical field between the spinneret and the collector, the polymer solution droplet at the needle tip will then undergo stretching and thinning into a fluid jet, as it travels towards the collector, followed by deposition onto the collector in the form of fibers. ${ }^{28}$ For each application, the nanofibers need to possess certain structural characteristics, thus for desalination, properties such as fiber diameter, morphology and membrane pore features are critical and they can easily be controlled by the tuning of electrospinning parameters.

In electrospinning, process optimisation is the first step for nanofiber production. Process optimization entails the selection of the appropriate polymer-solvent properties and process conditions in order to control the desired physical properties, such as fibre diameter, morphology, and mechanical strength of the resulting nanofibers. ${ }^{29}$

The selection of the polymer is the first step in the optimisation procedure and this depends mostly on the end application of the resulting nanofibrous membranes. For 

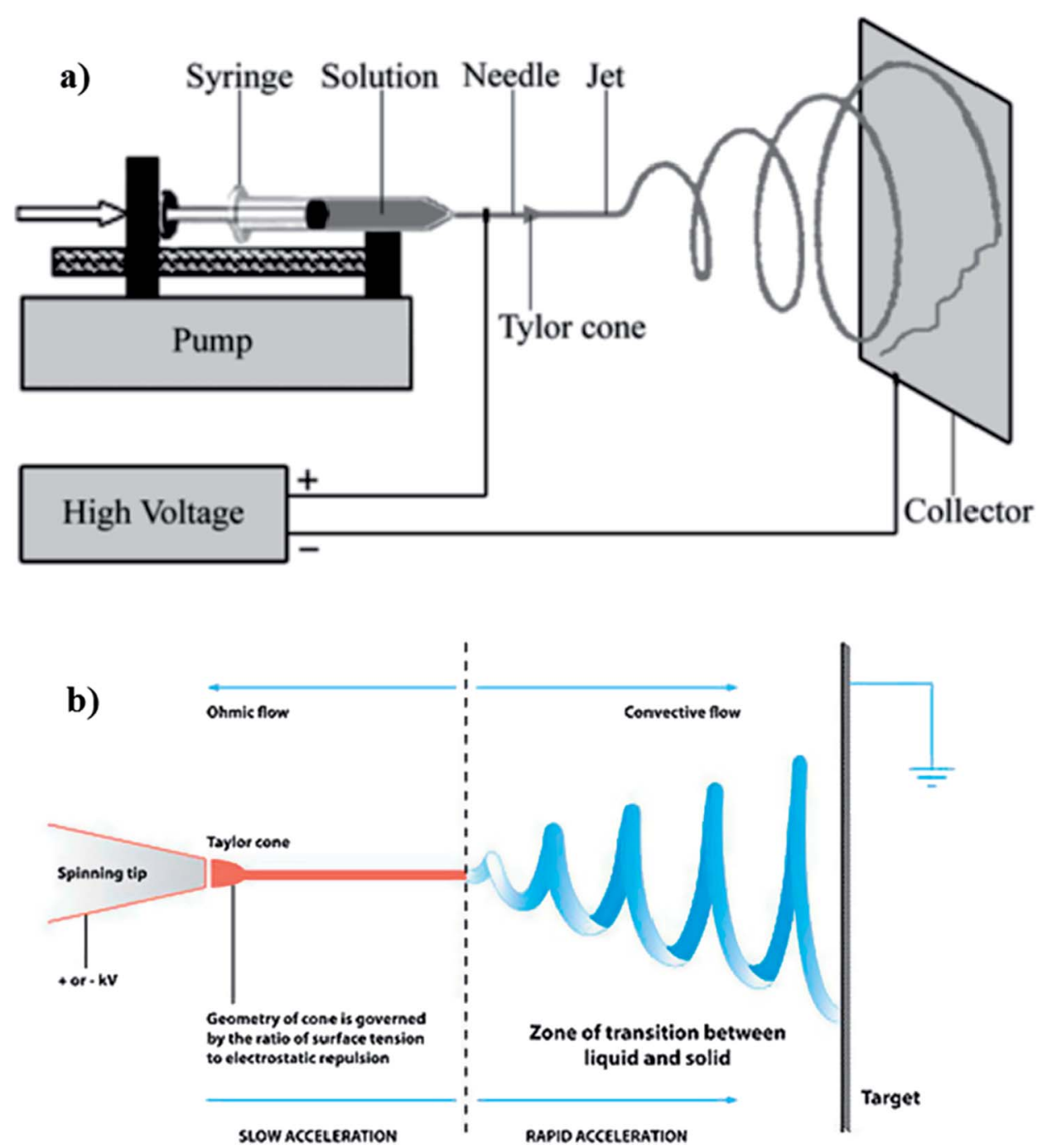

Fig. 6 Typical electrospinning set up showing (a) the basic components, and (b) the ohmic/convective flows regions of the jet flight. Reproduced with permission from ref. 28. Copyright 2010, Elsevier Science Ltd.

electrospinning of polymers, high molecular weight polymers are always preferred as they give enough chain entanglement during stretching of a polymer jet, which helps to minimise the formation of beaded nanofibers. The most common electrospun polymers for water-treatment use are PVDF, polysulfone (PSU), and cellulose acetate (CA). ${ }^{3,10}$

Solvents that readily dissolve the polymer without the need to be heated are highly preferred. A good solvent needs to have good conductivity, less surface tension, and sufficiently volatile. This facilitates fibre drying in order to avoid wet and defect free fibres deposited onto the collector. Solvents with boiling points that are too low will cause constant clogging of the needle due to rapid solvent evaporation (premature drying), while those with high boiling point will hinder fiber drying after reaching the collector, thereby resulting in wet fibers. A mixture of solvents, such as a binary solvent system is used to combine properties as a way of adding good properties of one solvent or to suppress the undesirable properties of the other solvent. For example, a chosen solvent may have poor conductivity and high volatility and therefore can be blended with a solvent that has better conductivity and low volatility. The volatility of a solvent is important as it will determine the rate of evaporation of polymer solution as the polymer jet travels toward the collector and deposited as nanofibers. Solvent evaporation is one of the main contributors towards porous fiber morphology, just like relative humidity. Although, not all solvents with low surface tension are good for electrospinning, a solvent with high conductivity and low surface tension are mostly suitable for any polymersolvent system. ${ }^{28}$ Such solvents offer high coulombic forces responsible for facilitating stretching of the polymer jet. Therefore, the surface tension can be overcome at a low voltage.

Different solvents have different surface tensions. ${ }^{28}$ Surface tension favors the formation of beaded fibers, because it offers resistant to the flow of polymer solution and stretching of a jet. The electrostatic force supplied by the applied voltage should overcome the surface tension for the jet to initiate. Usually, solvents with high surface tension are blended with those of lower surface tension and higher conductivity in order to adjust/ suppress the overall surface tension of the solution. 
A polymer solution is either conductive or dielectric. A dielectric polymer solution requires sufficient voltage to form a Taylor cone and initiate electrospinning and jet formation as only few charges are available in solution. A conductive polymer solution on the other hand, requires lesser critical voltage to initiate electrospinning, due to the availability of more free charges in the solution that are responsible for forming a Taylor cone. ${ }^{28}$ Solvents with good conductivity have high dielectric constant and this promotes high repulsive Coulomb forces (electric charge), thereby inducing stretching of a polymer jet. ${ }^{28}$ Studies show that the jet radius is inversely proportional to the cube root of the electrical conductivity, hence an increase in conductivity leads to a decrease in the fiber diameter. ${ }^{28}$ On the other hand, low conductivity causes weak electrical force (fewer ions), thereby resulting in insufficient elongation of jet and formation of beads due to jet break-up, forming droplets rather than fibers. Conductivity can also be enhanced by the addition of ionic salts to give finer, uniform and bead-free fibers. ${ }^{28}$

Viscosity of a polymer solution is directly proportional to the polymer solution concentration and molecular weight, i.e., number of chain entanglements among the polymer chains $\left(n_{\mathrm{e}}\right)$. This affects the degree of interaction between polymer chains and solvent and the polymer structure. ${ }^{30}$ Increase in concentration results in an increase in chain entanglement. Similarly, solution containing higher molecular weight polymer results in higher chain entanglement, add higher viscosity, when compared to one with the same concentration but lower molecular weight polymer. There is lack of continuous fiber formation at low viscosity, while high viscosity solutions are difficult to electrospin. Low viscosities offer high resistance to the formation of continuous fibers and to the elongation of polymer jet from the syringe, ${ }^{31}$ hence the formation of beads. Other factors that will affect the viscosity of a polymer solution (at the same concentration) are solvent ratios, temperature, and additives. Fig. 7 reports the polylactide (PLA) solution concentration dependence of fiber morphology.

The electric field created between the syringe needle tip and the collector induces charges onto the polymer solution droplet to form a Taylor cone. The charges are then transferred to the jet ejected from the needle and attracted to the collector, once the critical voltage has been reached. The critical voltage varies for each polymer-solvent system. High voltage increases the repulsive forces of fluid jet as more charges are introduced, which leads to increased pulling force and therefore, thin fibers are formed. The influence of voltage on fiber diameter is however, dependent on the solution concentration and spinning distance. As such, increase in fiber diameter with increase in voltage has also been reported..$^{30,32}$ This can be due to a number of factors: (i) an increased pulling of polymer solution volume out of a needle, although at a constant flowrate, ${ }^{33}$ (ii) an increase in the length of jet, which will in turn reduce the distance for whipping region (i.e., the convective flow region in
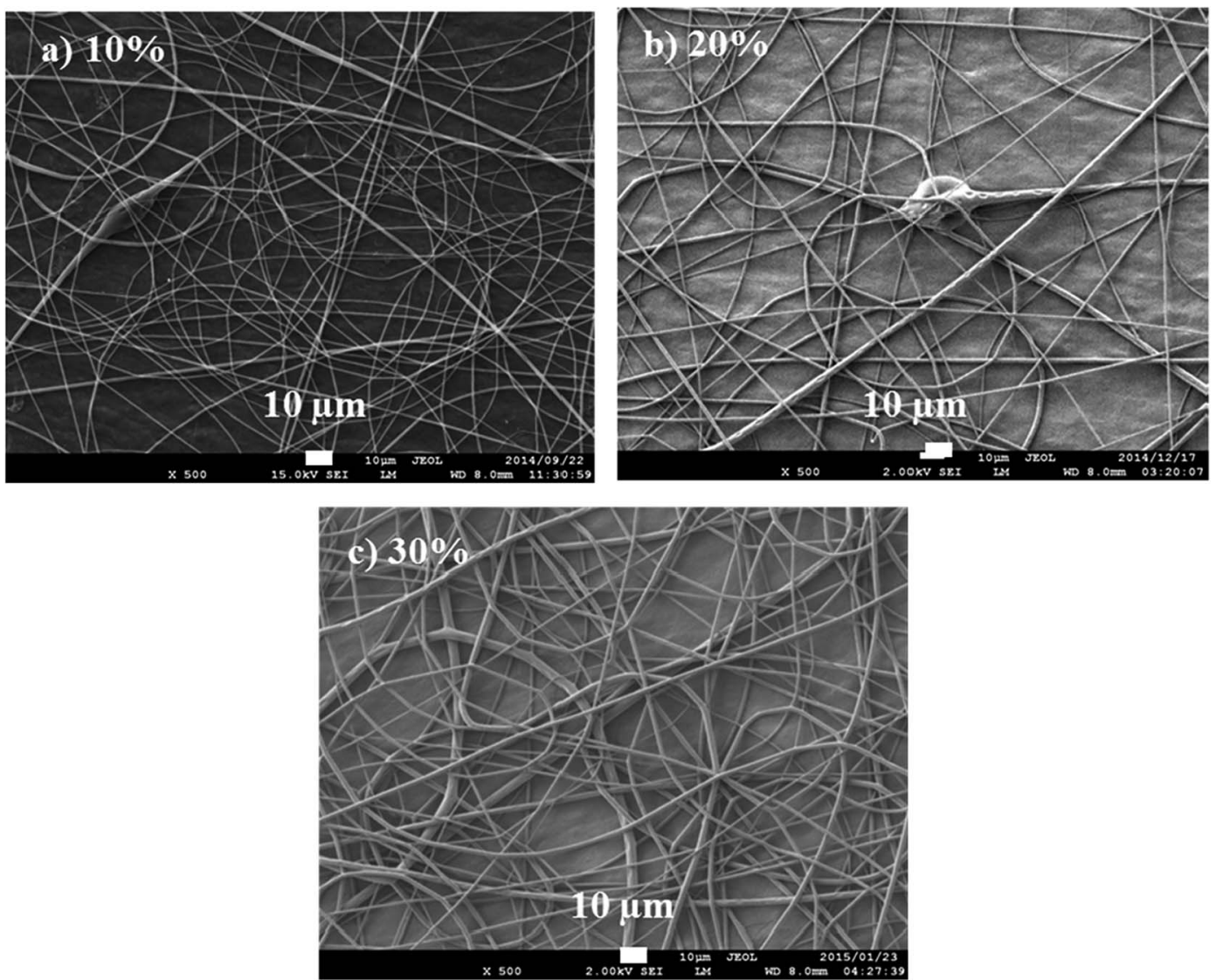

Fig. 7 Fibers produced from PLA solution concentrations of 10,12 , and $15 \mathrm{wt} / \mathrm{vol} \%$ spun at $20 \mathrm{kV}$ and $15 \mathrm{~cm}$ (spinning distance), and at a flow rate of $10 \mu \mathrm{L} \mathrm{min}{ }^{-1}$. 
Fig. 6(b)) responsible for further jet elongation and fiber diameter reduction, and (iii) slow jet flight because of weak acceleration of jet to the collector. ${ }^{34}$ There is also more solvent evaporation as the voltage increases. ${ }^{35}$ The reduction of fiber diameter continues until a critical voltage is reached, beyond which is the likelihood of bead formation. Optimization of voltage in order to obtain a stable Taylor cone is mainly a balance between the voltage, distance, and the flow rate. Low flow rates require low applied voltages, while high applied voltages are required when high flow rates are used. The effects of voltage and spinning distance on the fibre diameter were studied by using a $12 \mathrm{wt} / \mathrm{vol} \%$ PLA solution in $30: 70$ DCM : TFE. Voltages of $10,15,20$, and $25 \mathrm{kV}$ were tested with spinning distances of $10,12.5$, and $15 \mathrm{~cm}$. The flow rate was maintained at $10 \mu \mathrm{L} \mathrm{min}{ }^{-1}$. Fig. 8 reports the SEM images illustrating the effect of spinning distance and voltage difference on fiber diameter.
The spinning distance affects, directly the jet flight time, whipping distance and the voltage intensity. A short distance intensifies the electric field strength of the voltage and also results in a short jet flight. Spinning distance also controls the fiber diameter, morphology and bead formation. There should to be an optimum distance to allow for the solvent evaporation (fiber drying). Long or sufficient distances will result in beadfree and thin fibers. ${ }^{28,30}$

The rate of flow affects the rate at which the polymer solution is transferred from the syringe. For every flow rate, there is an optimum voltage to maintain a stable Taylor cone and jet (at a particular distance). An optimum flow rate maintains a balance between the polymer solution, leaving the needle, and the new volume being pumped. ${ }^{30}$ The balance of flow rate and pulling force of voltage determines the fiber diameter, porosity and geometry of fibers and the shape of Taylor cone. High flow

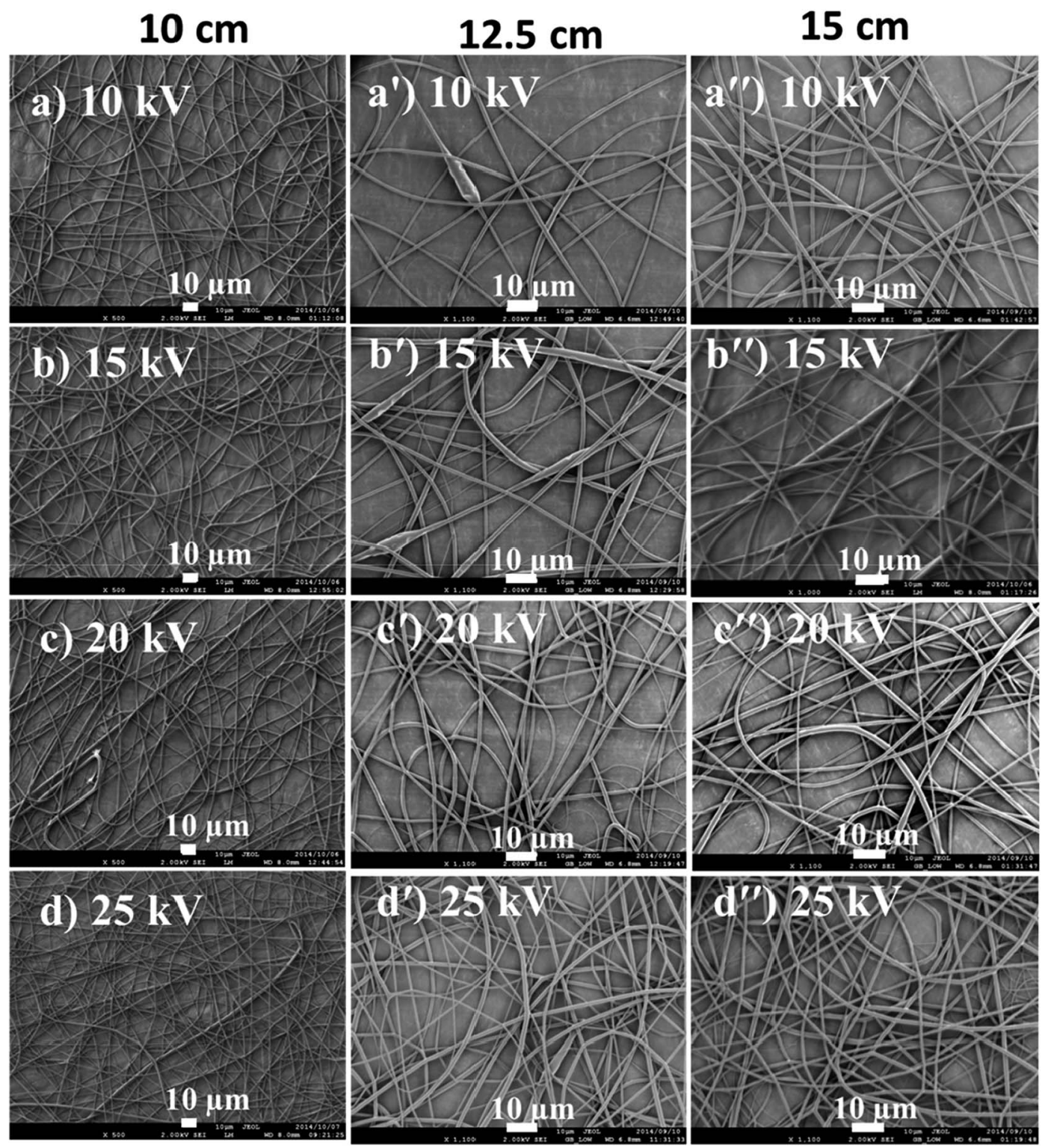

Fig. 8 PLA fibers spun from 12\% PLA/(30: 70 DCM/TFE) solution at distances of 10, 12.5 and $15 \mathrm{~cm}$ and voltages of $10,15,20$ and $25 \mathrm{kV}$. 

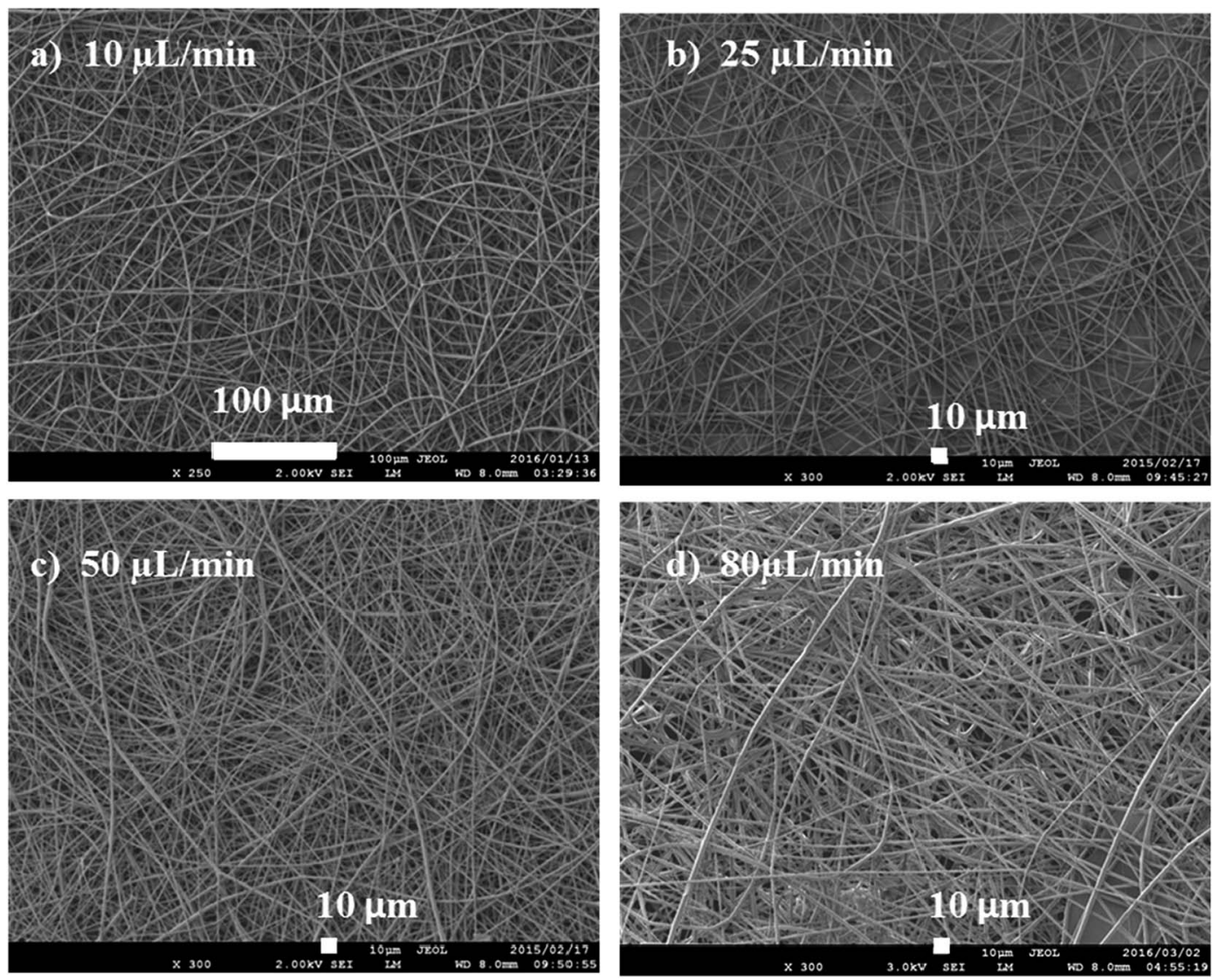

Fig. 9 Fibres spun using from a $15 \mathrm{wt} / \mathrm{vol} \%$ solution at flow rates of (a) 10 , (b) 25, (c) 50 , and (d) $80 \mu \mathrm{L} \mathrm{min}{ }^{-1}$.

rates are associated with large Taylor cone, resulting in large diameters and pore size (Fig. 9).

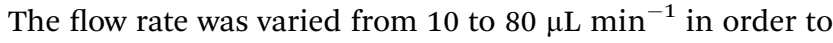
study its effect on fibre diameter. As shown in Fig. 9, the smallest fibre diameters were obtained at $10 \mu \mathrm{L} \min ^{-1}$, while the highest flow rates gave the largest diameters. As the flow rate increases (at constant voltage), more of the polymer solution is pulled from the needle, hence, resulting in thicker fibres. Increasing the flowrate leads to a bigger Taylor cone, and a longer, thicker jet. A longer jet produces a smaller whipping region, thereby limiting its further elongation and producing thicker fibres.

The ambient conditions mainly refer to temperature and relative humidity. Humidity affects fiber drying at the collector and this is dependent on the type of polymer-solvent system. For water soluble polymers, e.g., poly(ethylene oxide), poly(vinyl alcohol), etc., fiber diameter reduction, with an increase in humidity, is common. The reason being the fiber solidifying mechanism process, depending on the type of polymer. ${ }^{30}$ For binary solvent system, small circular pores in the fibers are likely to form in more humid conditions by a process called phase separation, when the more volatile solvent evaporates and followed by condensation during cooling. High humidity causes enlarged pores with high pore density. Low humidity has negative effects on the evaporation rate of the solvent and also causes clogging. ${ }^{35}$
As a conclusion, it is imperative that a stable Taylor cone is maintained once the optimum conditions for electrospinning are obtained, to ensure continuous and uninterrupted electrospinning. ${ }^{36}$ However, this remains a challenge for readily clogging polymer-solvent systems, where very highly volatile solvents, e.g., DCM are concerned. ${ }^{37}$ Through optimization, other factors, such as fibre diameter, can also be carefully controlled. The fiber diameter determines the resulting nanofibrous membrane properties, such as porosity, pore size, surface area, packing density and mechanical properties, which are vital for the end-use performance, such as water filtration applications.

\section{Electrospun polymeric nanofibrous membranes (ENMs) in desalination: reverse osmosis (RO) and membrane distillation (MD)}

Electrospun nanofibrous membranes are among emerging technologies for water treatment, due to advantages that include their abilities to produce high-quality water and, in most cases, with the consumption of less energy. They are therefore being developed into more functional membranes to meet most water treatment processes, particularly desalination. ${ }^{38}$ Nanofibers form a significant family of nanomaterials, 
and their use in the fabrication of novel composite membranes has evidently facilitated improvements in desalination performance.

\subsection{Water flux}

Also known as permeate flux; water flux can be optimized by tuning the electrospinning parameters. Low flux is often the result of low permeability, which results in membrane-surface fouling. ${ }^{39}$ The water flux $(J)$ is calculated as follows: ${ }^{40}$

$$
J\left(\mathrm{~L} \mathrm{~m}^{-2} \mathrm{~h}^{-1}\right)=\frac{\Delta g}{A \times t}
$$

where $\Delta g$ is amount of the permeate (L), $A$ is the effective membrane area $\left(\mathrm{m}^{2}\right)$, and $t$ is the duration of the operation $(\mathrm{h})$.

\subsection{Salt rejection}

Unlike particle filtration, the separation of dissolved salts (monovalent $\mathrm{NaCl}$ ) is a very complex process, and the ENM separation efficiency for such salts depends highly on the sizes of the membrane pores, which are primarily controlled by the fiber diameter through a direct relationship: $:^{\mathbf{8 , 4}}$

salt rejection $(\mathrm{SR} \%)$

$$
\begin{aligned}
& =\frac{\text { brine concentration }- \text { permeate concentration }}{\text { brine concentration }} \\
& =\frac{\text { brine conductivity }- \text { permeate conductivity }}{\text { brine conductivity }} \\
& =\frac{C_{\mathrm{f}}-C_{\mathrm{p}}}{C_{\mathrm{f}}} \times 100
\end{aligned}
$$

where brine concentration $\left(\mathrm{mg} \mathrm{L}^{-1}\right)$ refers to the feed $\left(C_{\mathrm{f}}\right)$, and the permeate $\left(\mathrm{mg} \mathrm{L}^{-1}\right)$ refers the product $\left(C_{\mathrm{p}}\right)$, which are obtained by measuring their conductivities.

\subsection{The use of ENMs in reverse osmosis}

Electrospun membranes (ENMs), on their own, are too porous to retain salt ions, as their pores are mostly larger than $100 \mathrm{~nm}$ and are distributed broadly; they are designed to operate on the microfiltration scale and are therefore suitable for MF separation when directly used (e.g., as pre-filters). Although highly permeable, their solute rejections are too low and they are also highly susceptible to fouling. However, ENMs can be applied to UF when further modified by a thin-film coating (by grafting or casting), and to NF or RO when coated through crosslinking or interfacial polymerization. ${ }^{41}$ Coating ENMs with thin-film layers is a hierarchical design strategy aimed at improving the functionalities and broadening the separation proficiencies of these membranes, thereby also improving their suitability for moredelicate separation processes, such as NF and RO. ENMs therefore require thin-film top coatings, which led to the development of TFNC membranes by grafting, crosslinking, or interfacial polymerization; ENMs are most commonly transformed into TFNC membranes by surface modification. ${ }^{\mathbf{4 2 - 4 5}}$

4.3.1 The development of ENMs as thin-film nanocomposite (TFNC) membranes for RO. Thin-film composite (TFC) membranes are nanofiltration-scale membranes specially designed for UF, NF, and mostly RO separation processes. They were first developed in the 1980s to replace the conventional cellulose membranes prepared by casting methods. TFCs are commonly prepared by interfacial polymerization (IP) and are therefore highly compatible with hydrophilic polymers such as PAN. Non-porous TFC membranes operate by a solutiondiffusion mechanism, as shown in Fig. 10, and offer superior properties, such as $\mathrm{pH}$ resistance, better permeabilities $(0.36$ $\mathrm{Lm}^{-2} \mathrm{~h}^{-1}$ bar $^{-1}$ ), good salt rejections of $99.8 \%$, and minimal fouling when compared to conventional cellulose phase-

\section{a) Hydrophilic nonporous RO membrane}

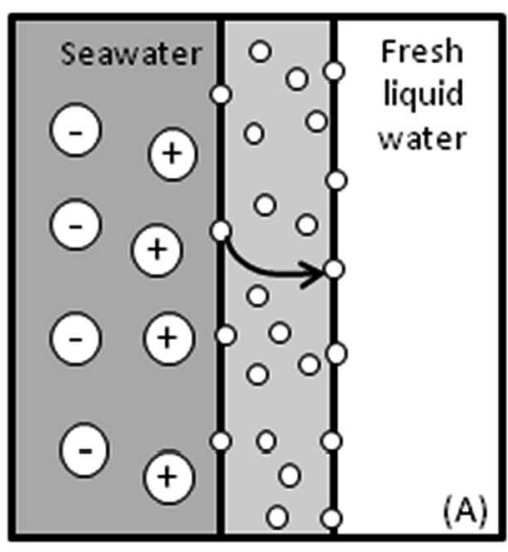

Scale $=\mathrm{nm}$

\section{b) Hydrophobic porous MD membrane}

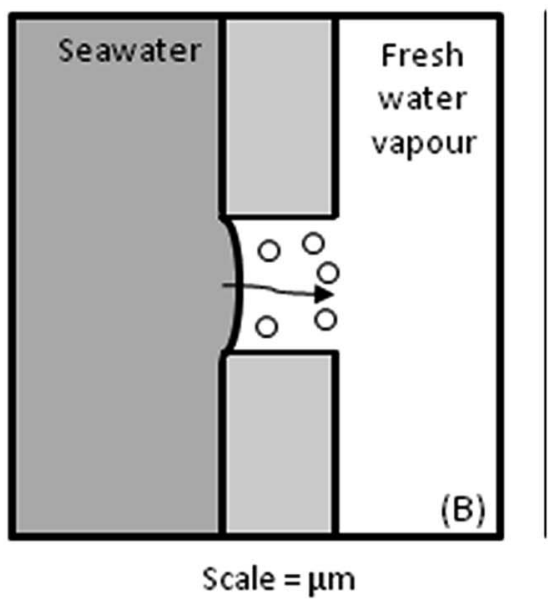

(†) cation $\left(-\right.$ anion $\mathrm{O}=\mathrm{H}_{2} \mathrm{O}$

Fig. 10 Transport mechanisms of a permeate through: (a) a nonporous TFC RO membrane and (b) a microporous MD membrane. Reproduced with permission from ref. 47. Copyright 2012, Elsevier Science Ltd. 


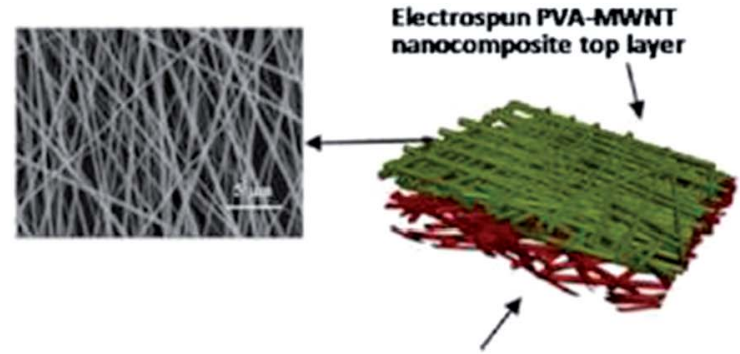

Electrospun PAN nanofibrous supporting layer

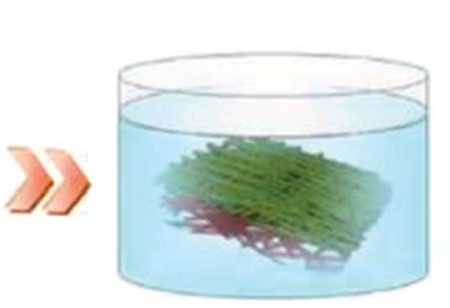

Step 1: Soak the PVA-MWNT/PAN double-layer mat in water/acetone mixture for solution treatment

Step 2: Add the crosslinking agent to the bath for PVA-MWNT nanocomposite top layer crosslinkine
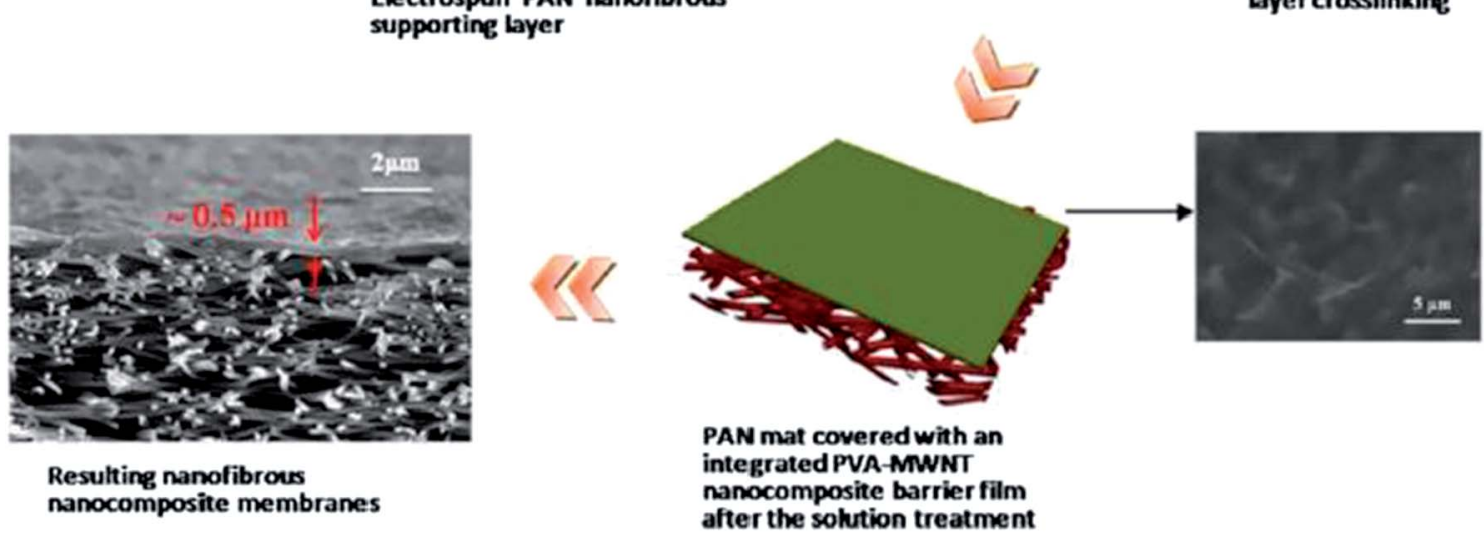

Fig. 11 Typical fabrication process for a TFC membrane based on an ENM substrate and a crosslinked PVA-MWCNT barrier layer. Reproduced with permission from ref. 48. Copyright 2013, Elsevier Science Ltd.

inversion membranes. The top coating is a densely cross-linked IP barrier or active layer where separation (salt rejection) takes place, as shown in Fig. 11. Each individual layer of a TFC membrane can easily be tailored to achieve optimal performance. The top and middle layers are the most fundamental and determine the overall performance of the membrane; hence they have undergone continuous modification in order to enhance performance, structure, and stability. ${ }^{8}$ TFC membranes consist of three layers: (a) an ultrathin dense polyamide (PA) material as the top hydrophilic active or barrier layer, which is coated onto (b) a conventional microporous phase-inversion membrane with pores $5-50 \mathrm{~nm}$ in size, which is coated onto (c) a nonwoven substrate, as depicted in Fig. 12.
Recent studies examined the possible use of a high-porosity ENM as a support for the nonporous top-layer coating as a strategy to further improve the performance of such composite membranes. The introduction of a fibrous microporous MF electrospun membrane (ENM) mid layer, as a replacement for the conventional sponge-like microporous UF mid layer produced by phase-inversion or casting (submicronsized pores) in a typical three-tier TFC membrane, led to the formation of the nano-based thin-film nanocomposite membrane (TFNC), which is one of the latest classes of novel desalination membrane. TFNC membranes are also regarded to be functionalized electrospun nanofibrous MF membranes as they can function in both NF and RO applications. ${ }^{42}$ The
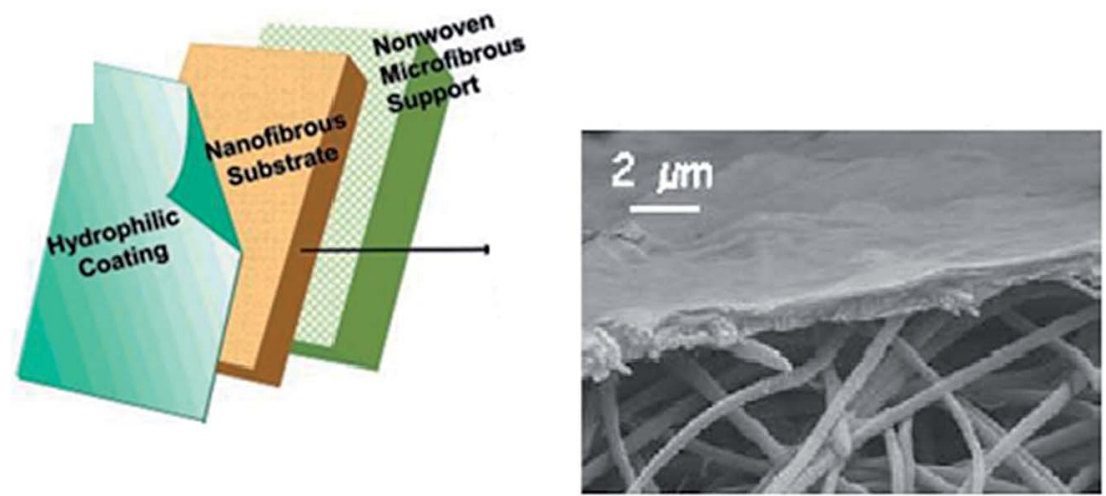

Fig. 12 A three-layered TFC membrane (the hydrophilic coating can be prepared by cast coating, cross linking, or interfacial polymerization (IP)) with a hydrophilic coating layer, a fibrous (TFNC/ENM) substrate, and a nonwoven substrate. The layers have average thicknesses of $0.2,40$, and $130 \mu \mathrm{m}$, respectively. Reproduced with permission from ref. 39. Copyright 2005, the American Chemical Society. 
introduction of an ENM therefore substantially enhances overall membrane performance due to its unique properties that include higher porosity $(10-20 \%$ higher than that of a phaseinversion membrane), low transmembrane pressure, and its interconnected pore structure. ${ }^{8}$ It is worth noting that ENMs have pore sizes on the MF scale, while those of phase-inversion membranes are on the UF scale. A highly porous ENM therefore substantially enhances the permeability of a non-porous thin film, which results in improved flux while maintaining good selectivity. However, ENMs face challenges. Their large pores lead to large numbers of TFNC-membrane defects, but at the same time thinner fibers effectively reduce pore sizes, resulting in increased selectivity at the expense of permeability. For processes such as RO that require hydrophilic membranes, ENMs face another challenge, as the electrospinning process tends to increase the hydrophobicity of the membrane; membranes in their electrospun nanofibrous forms tend to be more hydrophobic than the parent/bulk polymer or the membrane produced by phase inversion. ${ }^{8}$ Polymers such as PVA possess high surface tensions; therefore surfactants such as Triton X-100 are added to lower the surface tension. At the same time, the dense ultrathin nonporous structure of the top/active layer (with thicknesses ranging from 100 to $500 \mathrm{~nm}$ ) ensures good flux and selectivity while reducing hydraulic resistance. This demonstrates that the film should be as thin as possible to avoid hydraulic resistance. ${ }^{46}$ It is also worth noting that minimal fouling occurs with such a membrane, in the sense that solute particles adsorb and adhere less to the thin-film surface, while being prevented from penetrating into the underlying porous layers beneath the surface. ${ }^{39}$ Numerous improvements in top-layer performance have recently been reported, which include improved permeability and fouling behavior, while maintaining high levels of salt rejection., ${ }^{\mathbf{8}, 11,42}$

The concept of thin-film coated electrospun nanofibrous membranes was first applied to organic-solute rejection in water/oil separation. The use of TFNC membranes dates back to 2005 when a PVA/chitosan-hydrogel thin film was used as the top/active cast-coated layer on a chemically cross-linked (using glutaraldehyde) ENM substrate. The resulting TFNC ultrafiltration (UF) membrane was applied to the separation of colloidal solutions, such as water/oil suspensions. It is preferable that the cross-linking of the ENM has little or no effect on the membrane structure (e.g., porosity or fiber diameter) in order to retain its structural performance. In other words, crosslinking should result in the minimal degree of membrane shrinkage; for example, a $2 \%$ decrease in porosity is usually acceptable. ${ }^{8,39}$ UF membranes with top active thin-film layers produced by casting, cross linking, or phase polymerization, were later developed into NF membranes with top thin-filmcoating layers by the interfacial polymerization of polyamides onto the cross-linked electrospun nanofibers. Since then, interfacial polymerization has been commonly used to prepare membranes for the filtration of salt/seawater.

Interfacial polymerization involves the formation of a selective, highly cross-linked ultrathin polymer (usually polyamide) film with a thickness of about $300 \mathrm{~nm}$, and with a network structure at the interface between the two immiscible solutions (in which the two monomers are dissolved) following rapid chemical reaction. The parameters of this process determine the performance of the resultant TFNC membrane. Additives, such as piperazine comonomer, and their concentrations, play important roles in enhancing the flux, salt rejection, and strength of the thin-film selective layer. Due to the non-porous nature of the thin film, additives are incorporated in order to enhance permeability. ${ }^{10}$

The resulting nonporous thin-film top layer, however, offers resistance to the flow of liquid through the membrane, especially those prepared by IP. The less-porous or less-permeable (or macroscopically nonporous) TFNC barrier/thin-film layer therefore requires further treatment or modification using additives in order enhance permeability for increased membrane flux, while maintaining high salt rejection. Enhancing hydrophilicity is the most popular strategy for improving permeability, chlorine resistance, and fouling resistance. Another approach uses ENMs as porous thin-film layers, as discussed above.

4.3.1.1. Permeability enhancement. The incorporation of MWCNTs into a thin film can result in the development of microscopic or nanosized surface channels that promote water flow through the membrane, which increases both permeability and flux. The inclusion of nanoparticles in the top layer/thin-film results in the formation of what is referred to as a "nanocomposite coating layer" ${ }^{39}$ Other surface modifications that incorporate nanoparticles should, however, not hinder salt-rejection performance of the already nonporous membrane/active layer. Permeability has also been enhanced through the use of AgNPs, $\mathrm{TiO}_{2}$, $\mathrm{SiO}_{2}$, and zeolites, as well as organic additives, such as surfactants and solvents, while maintaining salt rejection. ${ }^{42}$

4.3.2 The roles of ENMs in TFNC membranes. The formation and adhesion of the top thin-film selective layer is also highly dependent on the structure and surface properties of the underlying ENM, namely morphology, fiber diameter, membrane pore size, surface chemistry, surface roughness, and polymer properties, such as hydrophilicity. ${ }^{10,42}$ The surface properties of the ENM also determine the overall performance of the composite membrane (e.g., salt rejection, permeability, fouling, mechanical, thermal and chemical integrity, and internal concentration polarization (ICP)). ICP is known cause masstransfer resistance in engineered osmosis (EO) processes. ${ }^{49}$ For example, hydrophilic polymers, such as PAN and CA, are effective supports for thin-film layers due to their effectively wetted and open pore structures with superior interconnectivities. However defects can form on the surface of the ultrathin selective layer due to the large ENM pores. ${ }^{8}$ The thinner the electrospun-membrane fibers, the better the performance, which is a consequence of reduced pore size; defects in the TFNC membrane are also reduced. ${ }^{50}$ Studies have used cast ultrathin nanofibrous cellulose fibers as the UF support for the top IP ultrathin selective layer. Thin UF cellulose nanofibers were prepared by casting; they had small $(\sim 50 \mathrm{~nm})$ pores and, hence, smooth surfaces; they were cast coated onto an MF PAN nanofibrous membrane counterpart with pores $\sim 650 \mathrm{~nm}$ in size. The obtained UF support layer exhibited a mean pore size of $20 \mathrm{~nm}$ and was about $100 \mathrm{~nm}$ thick, while maintaining the usual high porosities of the overall membrane that exceeded $70 \%$. The resulting TFNC was 
successfully used as an RO membrane for desalination. ${ }^{42}$ Thinner ENM fibers enhance salt rejection, but at the expense of water permeability. On the other hand, larger ENM pores favor high membrane permeability. In this regard, the porous ENM layer is required to be defect free and highly porous, chlorine resistant, and therefore highly permeable compared to the dense selective layer. Permeability increases with ENM thickness, which reveals that a relationship exists between the electrospun nanofibrous membrane morphology, particularly fiber diameter, and the performance of the TFNC membrane, as has been demonstrated. ${ }^{\mathbf{4 2}}$

4.3.3 ENMs as active layers for TFNC membranes. ENMs, with their highly porous structures, have been shown to be excellent mid-layer supports for top thin-film selective layers in highly permeable (high flux) TFNC membranes. However, ENMs have also been used as thin-film active layers themselves; ENM coatings have also been applied to UF oil/water separation. The PVA ENM melted during exposure to vapor and chemical cross-linking, resulting in a less-porous thin-film hydrophilic nanofibrous active layer, which was subsequently applied on a crosslinked PAN ENM substrate, as shown in Fig. 12. This was later further developed by a coating method that involved electrospraying droplets of a PVA solution that had been glutaraldehyde cross-linked. ${ }^{\mathbf{4 1}}$ Electrospraying is an electrospinning technique that is usually used to deposit thin films. Such studies are aimed at minimizing the disadvantages of some of the alternative thin film-methods (such as casting) in which the thickness of the top coating layer may be difficult to control using a casting solution, especially when applied onto a porous ENM substrate, as the cast-coating solution easily infiltrates into the ENM pores. Thickness is simply controlled by the duration of the electrospinning process. ${ }^{51}$ ENMs, as top thin film active layers, are known to exhibit superior permeabilities (although they are considered to be typical non-porous thin films); therefore they facilitate higher fluxes than thin films prepared by casting and interfacial polymerization. ${ }^{\mathbf{4 1}}$

4.3.4 Antifouling properties. Fouling occurs when unwanted filtered particles are deposited onto the surface or in the pores of the membrane and accumulate over time (as foulants), resulting in the degradation of membrane performance (flux). The problem propagates when the foulants come into contact with the active layer of the membrane. For hydrophilic membranes, fouling can be combated by minimizing contact between the foulants and the membrane. Therefore membranesurface modifications that enhance interactions with water and result in the increased affinity of water molecules are required. In other words, water molecules adsorb onto the membrane surface while solute particles are prevented from adsorbing. The membrane surface is thereby modified to be more hydrophilic, which is known as "hydrophilization". The active layer (of a TFC membrane) was originally thin-film surface coated in order to minimize membrane fouling. The thin film can be further hydrophilized by the use of hydrophilic additives to further reduce fouling. Although these membranes also show improved salt rejection, the membrane flux is often compromised. Strategies that mitigate fouling should, however, also favor salt rejection. ${ }^{11,21}$
4.3.5 Material selection for the ENM support layer in RO. Polymer membranes have extensively been used in RO processes, even though advances in other nanotechnology materials, including inorganic (ceramic), biomimetic (aquaporin), and CNT alternatives, have been applied to high-flux or highly permeable membranes. However, membrane design affects performance (selectivity, permeability, and fouling resistance) and its optimization is an ongoing process. To date, advances in membrane materials (among other membrane properties, such as structure and morphology) have resulted in increased RO efficiency that, in the long run, will result in economic viability. Therefore, material selection for the ENM support membranes in TFNC membranes for processes such as RO is also vital. ${ }^{1}$

The hydrophilic nonporous top-layer thin-film coating is more compatible with a hydrophilic nanofibrous ENM substrate. Hydrophobic substrates result in less contact between the membrane and the thin film during polymerization; hence surface treatment is required to increase its hydrophilicity. Polymers with good thermal and chemical resistance that are easy to process and biocompatible are preferred. The most commonly used examples include PVA, polyvinyl difluoride (PVDF), and polysulfone (PS). PVDF and PS also exhibit superior water stabilities. On the other hand, water-soluble polymers, such as PVA, are required to undergo crosslinking (with aldehydes) prior to application in order to impart water resistance/ stability, thereby preventing the dissolution of the membrane in aqueous environments. Thereafter, these membranes undergo shrinkage and water-stability testing, including water-solubility and water-absorbency testing, including swelling degree experiments, which determine the level of cross linking, in addition to mechanical testing. This testing regime determines the stability of an ENM membrane in an aqueous environment. PVA does not degrade under physiological conditions and has been used as both the coating and mid layers of a membrane. As a top layer, a cross-linked PVA hydrogel that forms a nonporous thin film was devised with added MWCNTs for permeability, which resulted in a high-flux membrane. Other hydrophilic polymers such as PAN, poly(ether ether ketone), polyimide (PA), poly(phthalazinone ether ketone), polypropylene (PP), and hydrolyzed cellulose acetate (CA) have also been used as ENM supports. ${ }^{7,39}$

4.3.6 Characteristics of ENM supports. An ideal ENM should have following characteristics: (i) superior porosities and pore interconnectivities that reduce ICP, (ii) improved water fluxes compared to other high-flux osmotic membranes that are performance-proven for engineered osmosis processes, and (iii) high fluxes and low transmembrane pressures resulting from high porosities and interconnected pores. As a mid-layer support (at high water cross-flow velocities in FO/PRO) they also enhance delamination resistance during operation. ${ }^{21}$ On the other hand, following characteristics: (i) reverse salt-flux leakage, (ii) salt flux increases rather than water/permeate flux, which is cause by damage to the active layer (e.g., due to long exposure to crossflow), the PA thin-film active layer is swollen due to hydrolysis (especially if a wetting agent is used), and $\mathrm{NaCl}$ salt is formed, which facilitates water absorption, (iii) the rigid PA structure gains as much as $28 \%$ of its weight due to 
exposure to water vapor, and (iv) the polymer chemistry and fabrication procedures need to be further optimization for improved performance, lead to membrane faults.

\subsection{Membrane fabrication for MD}

Membrane design and fabrication are of utmost importance for efficient desalination performance. Conventional membrane fabrication methods, such as phase inversion, produces membranes with large pores, high tortuosities, low hydrophobicities or surface roughnesses, and low porosities; consequently, they are not ideal for MD or any desalination process due to low fluxes and rejections. ${ }^{5}$ Likewise, ENM pores are also large; consequently, nanotechnology is required during their preparation, which involves the inclusion of nanoparticles/ nanofibers and high levels of modification. ${ }^{52-54}$ Since membrane technology plays a crucial role in desalination performance, new materials and fabrication methods for desalination membranes have been developed through progress in science and technology. As the aim of a desalination membrane is high salt rejection while maintaining high flux, ${ }^{\mathbf{8}}$ several factors that play roles in determining performance, such as membrane structure (porosity, thickness, and polymer/ membrane surface properties), need to be considered. Unlike RO, ENMs for MD applications can either be used as single (main membrane) layers or as support membranes (in multiple dual- or triple-layer systems). These are then applied in single layers as pre-filters that are placed upstream to enhance/protect the life of the more-delicate RO-filter membranes, or as direct filter membranes in UF or MD separations. As mid-layers they support the delicate top active layer in a multilayer composite membrane for NF or RO applications, and they can only be used independently in MD. For these reasons, ENMs are more promising for MD applications than other desalination technologies. ${ }^{\mathbf{8} 55-62}$

Since MD is a thermally driven process, single layers, as unsupported self-standing membranes, are more ideal for applications such as DCMD, in which they are not exposed to any harsh hydraulic/osmotic pressures. Although MD offers many advantages over other desalination processes, the membrane-fabrication methods, however, are continuously being improved in terms of structure and design in order to enhance the low permeation flux. ${ }^{21}$ For this reason, membrane design that particularly uses electrospinning, which is an emerging membrane-fabrication technique, needs further research. Membrane pore sizes can easily be adjusted by electrospinning, for example through the careful control of fiber diameter. ${ }^{63-65}$

4.4.1 ENMs as MD membranes, and materials used. The unique properties of electrospun membranes (high porosities, overlapping nanofibrous structures, and interconnected open pores) favor their use in MD applications. ${ }^{48}$ Hydrophobicity is the most basic requirement of an MD membrane; hence the first membrane-fabrication step involves the use of a hydrophobic polymer. ENMs have been used in membrane distillation due to their high porosities (void volume fractions) that augment the water-production rate (sufficient space for vapor transfer), membrane hydrophobicities (that render them less susceptible to wetting by feed water, thereby improving liquidentry pressure), high surface roughnesses, and low thermal conductivities (less heat conduction through the fibers). ${ }^{66}$ The production of membranes that are more hydrophobic than their bulk forms and those produced by other methods, such as phase-inversion and immersion-precipitation, is another advantage of electrospinning for MD applications. ${ }^{3,48,67}$ Hydrophobic polymers commonly used in MD include PVDF-HFP, PP, PES (modified), polytetrafluoroethylene (PTFE), and polyvinylidene fluoride (PVDF). For example, PEI is mostly used on the laboratory scale (i.e., for electrospinning or phase-inversion) as it easily dissolves in solvents, while other polymers are produced commercially. PVDF and its co-polymer, PVDF-HFP, have successfully been electrospun for DCMD. They are the most chemically, thermally, and mechanically stable polymers, and are also commonly used in electrospun membranes for MD. In addition, copolymers of PVDF (i.e., PVDF-HFP) have higher hydrophobicities than PVDF itself..$^{\mathbf{2 0 6 8 - 7 0}}$ The high surface roughnesses of ENMs are well suited to $\mathrm{MD}$, which evolved into membranes with enhanced hydrophobicities compared to those prepared by phase inversion. For example, the contact angle of a hydrophobic polymer, such as PVDF, is about $90^{\circ}$ as a flat membrane; this value increased to $138^{\circ}$ when the membrane was electrospun. Electrospinning has also been used an alternative technique for instilling superhydrophobicity in hydrophobic (or low surface-energy) polymers. ${ }^{67,71,72}$ In addition, electrospun neat polymers can be made superhydrophobic by further increasing or modifying its surface roughness or chemistry by incorporating nanoparticles (e.g., Ag, hydrophobic $\mathrm{SiO}_{2}, \mathrm{TiO}_{2}$, and clay). As a result, the contact angle can be as high as $158^{\circ}$ when AgNPs are added to the ENM-polymer matrix. However, the addition of some particles, such as clay, can cause the fiber diameter to increase. ${ }^{73-84}$ The performance and some properties of such membranes are shown on the Table 1. MD membranes are constructed using two or three tiers of hydrophobic-hydrophilic membrane layers, unlike in the single unsupported hydrophobic-layer design. The thin electrospun hydrophobic membrane, as the top active layer facing the feed side, is highly porous and is responsible for separation and mass transfer. The second hydrophilic-support layer enhances permeate flux with its wettable nature, and also contributes to reducing masstransfer resistance as well as heat-transfer or conductivity of the top layer. The second layer is also electrospun, but with a hydrophilic polymer, such as PAN, PVA, or N6. ${ }^{6}$ Electrospun nanofibrous membranes have therefore successfully replaced conventional microfiltration membranes for MD. ${ }^{69}$ The use of electrospun nanofibrous membranes (ENMs) in MD dates back to 2008, when PVDF membranes were used for the production of drinking water by AGMD. ${ }^{3}$ However, the need to improve flux and water quality led to membrane modifications that increased hydrophobicity. In addition to selecting the hydrophobic polymer, hydrophobicity can be enhanced by modifying the surface with additives, such as nanoparticles (NPs). For instance, incorporation of clay NPs into a hydrophobic polymer, such as PVDF, further enhances its hydrophobicity 


\section{RSC Advances}

Review

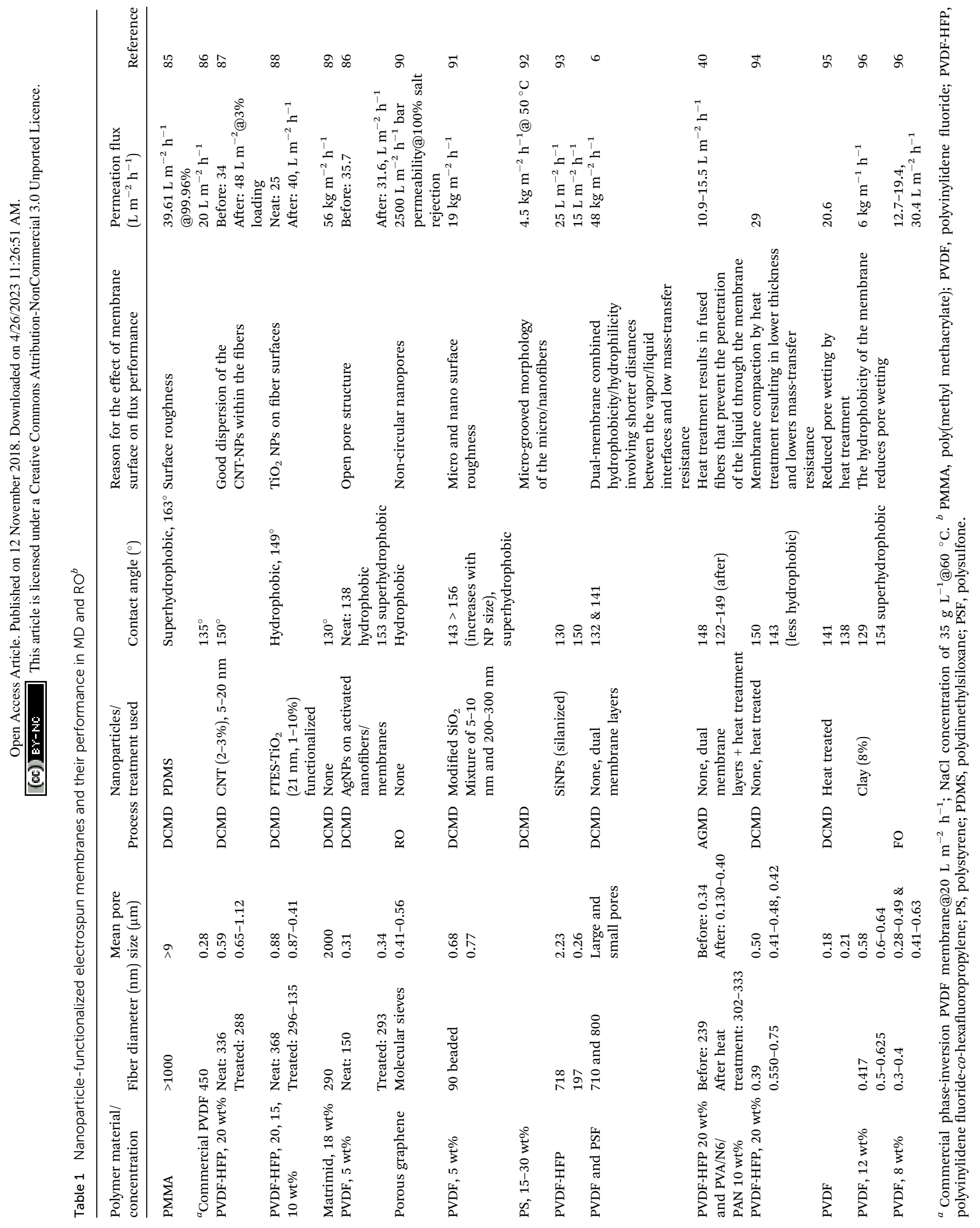


(superhydrophobicity), thereby improving flux by preventing or reducing pore wetting. ${ }^{8}$ In addition to hydrophobic-polymer electrospinning, recently developed hydrophobic materials, including polymers that are known to possess superhydrophobic properties, have also been electrospun. The produced membranes are known to offer high permeate fluxes (of up to $85 \mathrm{~kg} \mathrm{~m}^{-2} \mathrm{~h}^{-1}$ ), and include polyazoles and their copolymers, but require solution heating.

4.4.2 Design and structure of ENMs for MD. Not so long ago, there were an insufficient number of membranes specifically designed for MD; consequently most studies have focused on the fabrication of membranes for this process. Most attention has been given to membrane optimization such as surface modification. Consequently, high porosity, and highly hydrophobic (e.g., superhydrophobic PVDF) membranes have been developed in recent years as a consequence. ${ }^{86,97,98}$ For MD, membranes with high hydrophobicities, low tortuosities, and high thermal and mechanical stabilities, have been developed. Although these are characteristics desired in MD membranes, challenges remain that include improving salt rejection while maintaining permeate flux, and membrane thickness at the cost of mechanical strength.

Since MD membranes can be fabricated in single, dual, or triple layers, they have simpler designs compared to those used in RO and NF. As the required pores are in the microfiltration/ ultrafiltration range, MD membranes can be produced in a single process, such as electrospinning; i.e., without an additional selective-layer coating by interfacial polymerization, as used in the fabrication of TFNC membranes for RO. Therefore unsupported single-layered membranes (i.e., self-sustained membranes) only consist of a hydrophobic layer when electrospun. As they are self-supporting, they are required to be mechanically and chemically stable. MD also uses multi/dual layers in which the top hydrophobic layer is coupled with a hydrophilic backing layer. Therefore, recent studies use a combination of hydrophobic and hydrophilic layers to form a bilayer of composite MD membrane, i.e., the hydrophobic membrane is modified by introducing a hydrophilic support layer. The underlying hydrophilic layer, together with its high porosity, aids in enhancing the permeate flux of the top hydrophobic membrane, while maintaining high salt rejection. Ray et $a l .{ }^{99}$ produced a dual layered membrane consisting of electrospun polypropylene (PP) as top hydrophobic layer and PVA as $2^{\text {nd }}$ hydrophilic layer form a composite membrane. The added surfactant helps to form a membrane with thinner uniform fiber diameters and thus smaller pore sizes and higher surface area. Thus the hydrophilicity of bottom layer enhanced by surfactant formed an overall membrane with higher water adsorption, therefore higher flux, due to the formation of nanochannels that resulted in high pore volume. ${ }^{99}$ Single layers with or without a backing layer have also been employed in MD. ${ }^{21}$

4.4.3 ENM characteristics that affect MD performance. MD performance, as with other membrane desalination processes, depends on membrane structure in terms of thickness, porosity, pore properties (mean pore size and distribution), and geometry. Unlike RO TFNC membranes, MD requires microporous hydrophobic membranes that are thermally and chemically resistant. The development of membranes that function as interfaces between two fluid phases, while preventing their interdispersion and maintaining high vapor mass-transfer rates, as well as resistance to liquid intrusion through membrane pores, are major MD achievements. ${ }^{\mathbf{1 0 0}}$ The pores of electrospun membranes are smaller than those of conventional membranes, but are sufficiently large to ensure vapor permeation through the membrane (with the benefit of less fouling), which makes them more suitable for MD processes, unlike the membranes required for RO. ${ }^{\mathbf{6 8 , 1 0 1}}$

4.4.3.1 Thickness of the active layer. The thickness of an electrospun membrane is tailored during fabrication by adjusting the duration of the electrospinning process. Thickness also plays an important role in the overall membrane performance, and the membrane is usually considered to be thin when it is less than 400 $\mu \mathrm{m}$ thick. For example, while the mass/vapor-transfer resistance of a membrane increases with increasing thickness, the permeate flux decreases due to increases in mass-transfer resistance that result from the longer transport pathway. ${ }^{21}$ While thinner membranes can be used in thermal processes, it is worth noting that thicker membranes are beneficial for thermal resistance and mechanical stability in MD processes. The required thickness also depends on the membrane support and type of feed solution (salt concentration). However, wetting increases with decreasing membrane thickness due to liquid penetration through the pores. The effective thickness of the support layer is defined as: $S=t \tau / \varepsilon$, where $t$ is the thickness, $\tau$ is the tortuosity, and $\varepsilon$ is the porosity of the support membrane; this is also known as the effective diffusive path and determines the mass-transfer resistance, which needs to be lowered by lowering thickness and increasing porosity. ${ }^{\mathbf{1 0 2 , 1 0 3}} \mathrm{A}$ study by Essalhi and Khayet ${ }^{5}$ used electrospun PVDF membrane as a single hydrophobic layer membrane, with optimized thickness. As the thickness is one of the membrane properties that affect the membrane performance such as flux and thermal resistance, the thickness was adjusted at varying feed concentration and temperature, in order to investigate its effect on MD performance. Although thickness is beneficial for energy efficient for MD process, thinner membranes however, achieve higher permeate fluxes due to increased permeability, as flux is inversely proportional to membrane thickness, their larger pore sizes, higher porosity and lower tortuosity. ${ }^{5}$ Another interesting study by Khayet et $a .^{6}$ demonstrated how the membrane flux can be enhanced substantially, by optimizing the thickness of the underlying layer rather than introducing an underlying hydrophilic layer below the hydrophobic top layer. Thus the increased flux with the reduced thickness was due to increase in membrane pore size and porosity to facilitate easy water transport through the dual layered membrane. ${ }^{6}$ For pressure-driven processes, reducing the membrane thickness, in an attempt to increase the permeate flux, should not sacrifice the mechanical integrity of the membrane due to the high pressure. A lack of membrane-thickness uniformity remains a challenge for ENMs. ${ }^{21,23}$ Therefore, increases in thickness are highly beneficial for mass-transfer resistance, good mechanical stability and strength, and permeability.

4.4.3.2 Membrane pores. The most important characteristic of the MD-membrane pores is dryness, which can be successfully achieved without impacting permeability through 
the use of hydrophobicity and small pores. ${ }^{\mathbf{1 0 4}}$ Adjusting the sizes of the membrane pores during electrospinning involves tuning certain parameters, such as the composition of the polymer solution, humidity, and the needle speed (horizontal translation). As the feed water should not penetrate into the membrane pores, the operating or hydrostatic-process pressure must not exceed the liquid-entry pressure (LEP) of the membrane. LEP refers to the minimum transmembrane pressure, or the pressure at which the liquid can enter the membrane pores, and depends on the maximum pore size, shape, and hydrophobicity. Since the LEP and the feed concentration are directly related, this value may be higher for feed solutions with high $\mathrm{NaCl}$ contents, such as seawater, due to increased surface tension. It is therefore preferable that the hydrostatic pressure is lower than the LEP to avoid pore wetting. Hence higher LEP values can be obtained and maintained with higher hydrophobicities, smaller pores (that do not affect the membrane permeability), and thicker membranes. Decreases in the LEP value resulting from increased in the thickness of the hydrophilic layer in a duallayer membrane are attributed to increases in the inter-fiber space. 5,21,68 However, along with membrane fouling, scaling and membrane wetting can still occur. Therefore, the hydrostatic pressure must be lower than the LEP, and can be calculated using the Laplace equation:

$$
\mathrm{LEP}=\frac{B \gamma_{\mathrm{L}} \cos \theta}{d_{\max }}
$$

where $B$ refers to the geometric structure of the membrane pores ( $B=1$ for cylindrical pores), $\gamma_{\mathrm{L}}$ is the surface tension of the salt solution, and $\theta$ is the contact angle between the membrane and solution surface. ${ }^{21,22}$

4.4.3.3 Pore size and distribution. Pore size refers to the mean pore size, while distribution refers to the variety of pore sizes within the membrane, particularly those prepared by electrospinning. Electrospun nanofibrous membranes usually have pores around $0.1-1 \mu \mathrm{m}$ in size; therefore these MF pores are suitable for MD use. Large pores provide higher permeate fluxes; however they should be small enough to prevent water penetration, while maintaining good permeability. ${ }^{92}$ A masstransfer mechanism exists for each pore-size distribution. The pore size distribution (PSD) is mainly affected by the diameters of the membrane fibers because pore size is inversely proportional to fiber diameter. The pores in an MD membrane should vary little in size. Narrow pore-size distributions (associated with thin fibers) and small pores of about $0.60 \mu \mathrm{m}$ in size, or less, are preferred. A larger PSD leads to pore wetting/ penetration, which allows solutes from the feed to pass through the membrane (low rejection). Unfortunately only a $25 \%$ increase in pore size during operation can be accommodated. In MD, the flow of vapor is governed by mechanisms that depend on the sizes of the pores within the membrane; these include Knudsen diffusion, Poiseuille/viscous flow, and molecular diffusion. ${ }^{64,65} \mathrm{Su}$ et al. ${ }^{69}$ observed that a pore-diameter distribution for their electrospun PVDF mid-layer/support membrane centered at about $390 \mathrm{~nm}$ gave the best DCMD performance. Better pore characteristics and structure are indicated by a narrower pore distribution and a more focused (sharper) maximum peak. Pore-size distribution also affects water quality in terms of conductivity; narrower pore-size distributions provide higher water quality as fewer ions permeate through the membrane. Commercial membranes have narrow pore-size distributions; hence they display better pore properties than ENMs. For example, a commercial PTFE membrane has a pore-size distribution of $0.1-0.35 \mu \mathrm{m}$, compared to that of an electrospun PVDF $(0.2-0.8 \mu \mathrm{m})$ with a $220 \mathrm{~nm}$ average fiber diameter. However, it is worth noting that the ENMs from co-polymers often exhibit better performance compared to membranes with narrower pore-size distributions. The PVDF co-polymer resulted a membrane with better performance than commercial membranes due to its narrower pore-size distribution, thinner fibers, and better contact angle (hydrophobicity); consequently, they are preferred for MD applications over PVDF ENMs. ${ }^{69}$ Fig. 13 displays nanofibrous membranes modified with AgNPs and their corresponding pore-size distributions. Hydrophobicity or surfaceroughness modifications resulting from the incorporation of nanoparticles affect the pore sizes and the pore-size distributions of the membranes. Fig. 13 shows that the once bare fibers in panel (a) become covered with nanoparticles, which reduces the sizes of the pores, as seen in panel (b). However, panel (c) reveals that full coverage of the membrane with a layer of particles leads to complete pore blockage, which often has a detrimental effect on membrane performance due to decreased water permeability and, consequently, reduced flux; it well established that pore-size distribution affects water flux. ${ }^{\mathbf{8 6}}$ Surface modifications therefore need to be effective or optimized in such a way that the sizes and distributions of the pores are not affected. For example, the pore-size distributions of the modified and unmodified membranes shown in Fig. 14(a and b) that correspond to the images in Fig. 13(a and b) are quite similar, and compete with those of the commercial membrane shown in panel (c). The narrow pore-size distribution is unaffected by the inclusion of AgNPs, unlike the broad pore-size distribution that results from blocked pores following ineffective surface modification, as shown in Fig. 14(c). Other membrane-surface modifications, such as heat treatment, also result in pore sizes that remain narrowly distributed, however reduced fluxes are observed. ${ }^{\mathbf{4 0 , 1 0 5}}$ Although the incorporation of NPs seems to increase the fiber diameter more than heat treatment, the former membranes exhibit enhanced performance compared to the latter..$^{5,67,75,106-108}$

4.4.3.4 Porosity or void-volume fraction. Like membranes used in other desalination processes, porosity is required to be as high as possible for adequate vapor transport, and low thicknesses and tortuosities are required. Porosity, which is the void-volume fraction of the membrane, can either be surface or volumetric porosity. ENMs for MD have large porosities (of up to 90\%), enhanced permeation fluxes (permeabilities), as well as large surface areas for evaporation, and numerous pore channels for diffusion. High porosity also means more voids; hence the presence of low-conductivity air spaces help to increase the thermal resistance (heat efficiency) of the membrane. The porosity should be neither too high nor too low. A high porosity 
detrimentally affects the mechanical properties of the membrane. MD membranes with porosities in the 35-93\% range have been prepared; for example, the porosity of an electrospun PVDF membrane is $79 \%$, while that of a commercial PVDF is $62 \% .^{21,24,109}$ Porosity can be determined gravimetrically as the volume of the pores divided by the total volume of the membrane: ${ }^{40}$

$$
\rho=\frac{\left(W_{1}-W_{2}\right) / D_{\mathrm{e}}}{\left[\left(W_{1}-W_{2}\right) / D_{\mathrm{e}}\right]+W_{2} / D_{\mathrm{p}}}
$$

where $\rho$ is the porosity, $W_{1}$ is the weight (g) of the wet membrane (soaked in ethanol), $W_{2}$ is the weight of the dry membrane, and $D_{\mathrm{e}}$ is the density $\left(\mathrm{g} \mathrm{m}^{-3}\right)$ of the wetting liquid (e.g., ethanol).

Tortuosity refers to the lack of straightness of the pore channels; the higher the tortuosity, the lower the permeability. A cylindrical channel has a tortuosity that is close to unity and is desirable for MD. ${ }^{\mathbf{1 1 0 , 1 1 1}}$
4.4.3.5 Hydrophobicity. The material composition (polymer type) and the geometrical structure of the surface determines the hydrophobicity (or surface energy) of the membrane. The role of a hydrophobic membrane surface in MD is to prevent membrane wetting or pore penetration by the liquid phase (feed water), thereby facilitating the penetration of only the vapor phase. ${ }^{\mathbf{1 0}}$ Conventional MD membranes are typical MF membranes prepared by phase-inversion using hydrophobic polymers such as PP, PTFE, and PVDF, with contact angles of $\sim 138^{\circ}$ that are higher than those of flat-fabricated PVDF membranes $\left(128^{\circ}\right)$, and have good chemical and thermal properties. The electrospinning of these polymers affords membranes with better hydrophobicities owing to the surface roughnesses by the nanofibers; consequently, low contact between the solid and liquid surfaces is achieved, which, in turn, leads to a higher contact angle (CA). Polymer hydrophobicity can also be enhanced through modification with hydrophobic additives or low-surface-energy materials, such as
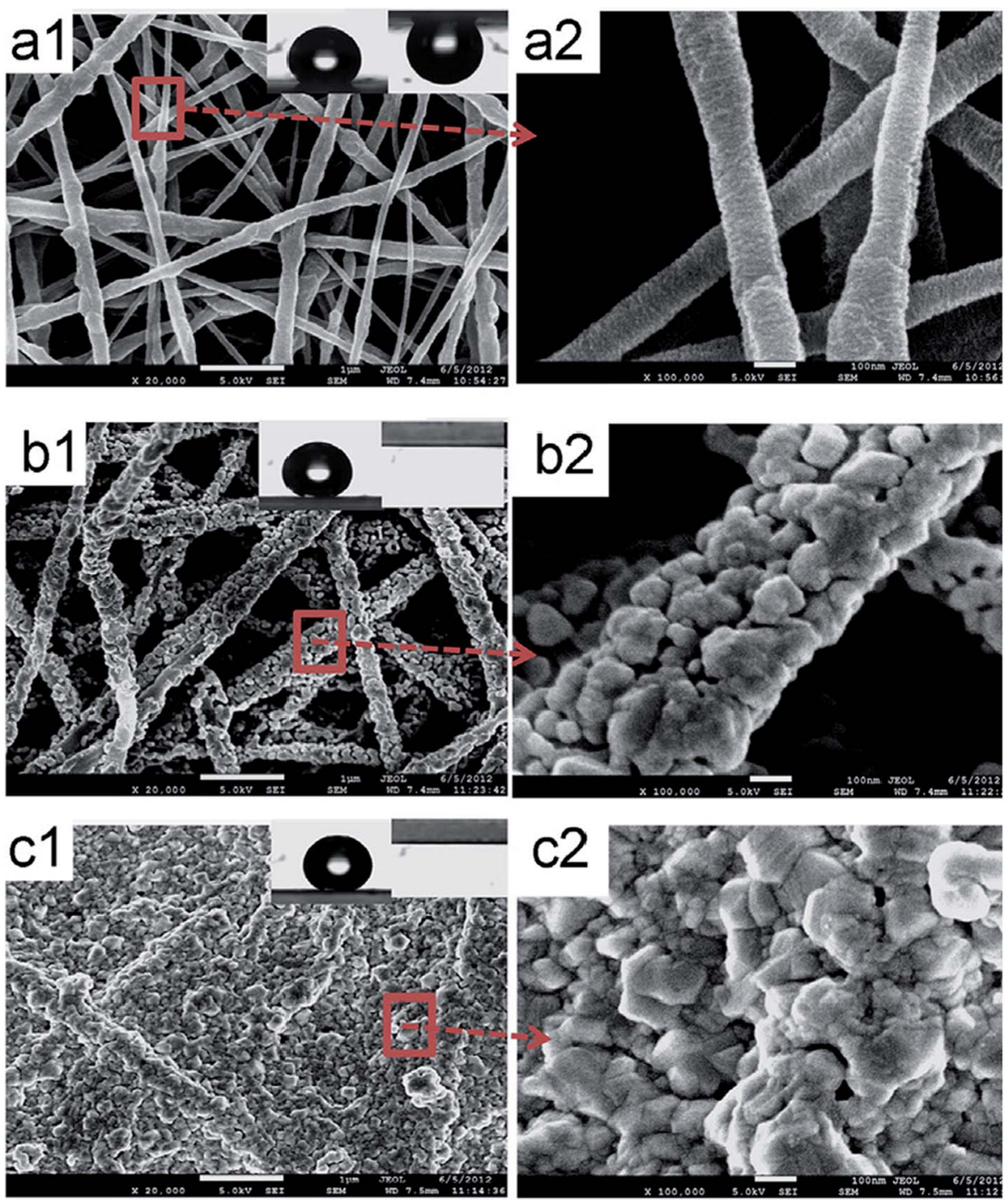

Fig. 13 SEM images of superhydrophobic nanofibrous membranes with and without the incorporation of AgNPs. (a) Before modification (pristine fibers), (b) after fiber coating (AgNPs are deposited onto individual fibers), and (c) after membrane-surface coating (AgNPs cover the entire membrane surface). Reproduced with permission from ref. 86. Copyright 2012, Elsevier Science Ltd. 


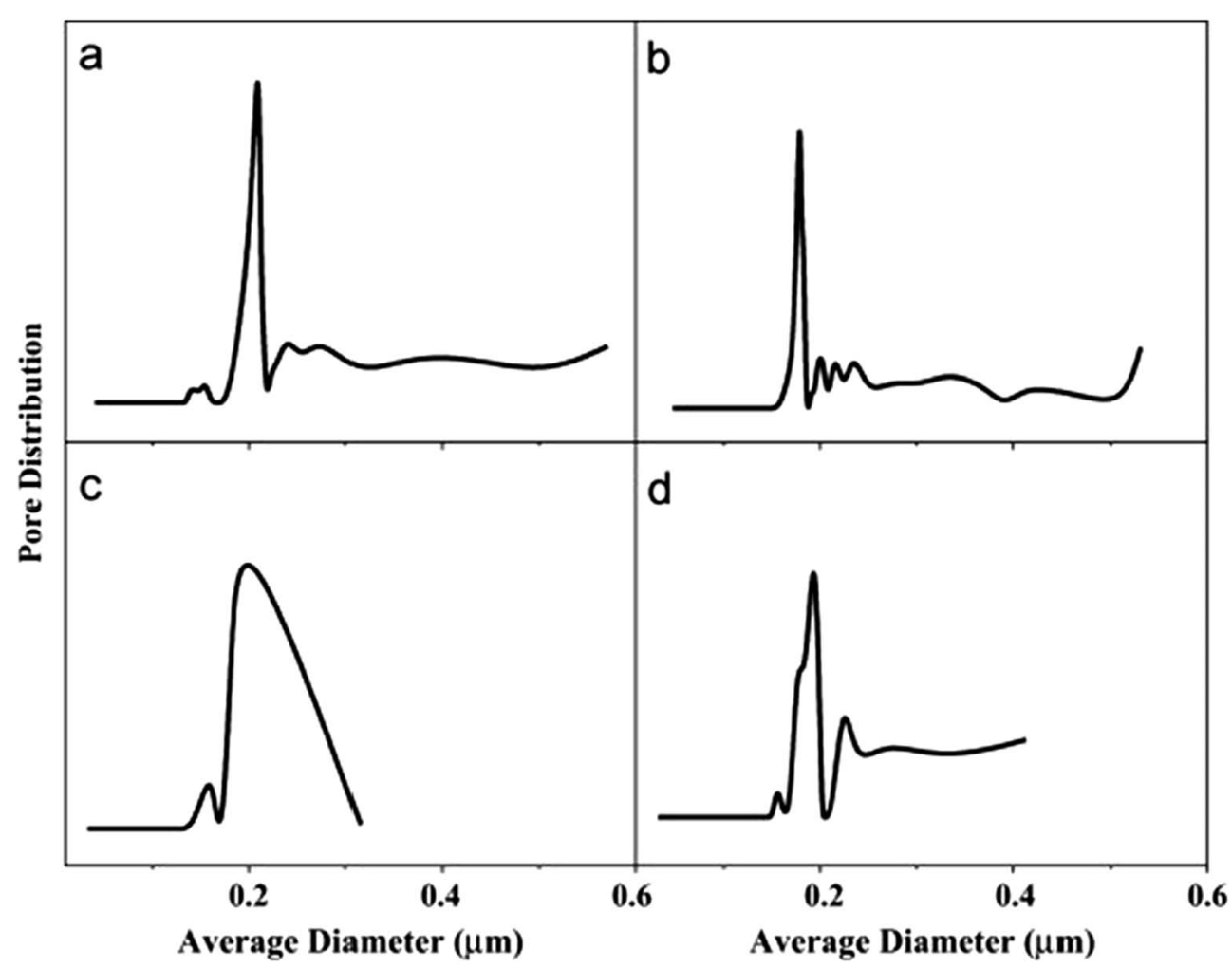

Fig. 14 Pore-size distributions of the electrospun PVDF membranes depicted Fig. 13: (a) unmodified, (b) fully modified (individually coated fibers), (c) coating only on the membrane surface, and (d) a commercial PVDF membrane for comparison. Reproduced with permission from ref. 86. Copyright 2012, Elsevier Science Ltd.

hydrophobic NPs, in the polymer matrix, molecular and functional-group modifications, or by introducing or increasing surface roughness. The water contact angles of these membranes before and after surface modification are depicted in Fig. 15. Surface modification techniques, such as plasma treatment and layer-by-layer assembly, can also be used. ${ }^{112-114} \mathrm{~A}$ superhydrophobic surface is obtained by increasing the surface roughness of two materials with different hydrophobicities; materials with high contact angles $\left(>150^{\circ}\right)$ are considered to be superhydrophilic or ultrahydrophobic; i.e., a water droplet on the polymer surface will exhibit a low sliding angle or contact angle hysteresis of less than $10^{\circ}$, which is the difference between the contact angles of the front and back edges of the water droplet. ${ }^{113,115}$ Hydrophobicity enhances membrane performance in $\mathrm{MD}$, including improved salt rejection and reduced pore wetting. ${ }^{20,116,117}$ Hydrophobicity affects the membrane-filtration efficiency; hydrophobicity is the result of membrane-surface roughness, while hydrophilicity is associated with smoothness. The ENMs are rendered rough due to air pockets formed between the fibers, which lead to increased hydrophobicity compared to the unspun membrane. Hydrophobicity, which is determined by contact-angle measurements, is required to be as high as possible for the DCMD process, and membranes or polymers with higher contact angles are usually preferred for MD applications. PVDF-HFP, with a high contact angle of $128^{\circ}$, provided more satisfactory results than the commercial PTFE with the lower contact angle of $115^{\circ}{ }^{8,69}$
4.4.3.6 Thermal properties of membranes. The membrane material should have a low thermal conductivity as well as good thermal resistance. High membrane porosities are associated with low thermal conductivities, as the presence of more air within the pores helps to reduce heat transfer by conduction. Another method involves the use of multilayers (dual layers with a hydrophobic top layer on a hydrophilic layer/support), in which the thickness of the support helps to lower heat conductivity.

4.4.3.7 Post-electrospinning heat treatment. Hot pressing is the most common heat treatment for electrospun desalination membranes, and involves membrane compaction by pressing between two plates at a temperature above the glass-transition temperature $\left(T_{\mathrm{g}}\right)$, but below the melting temperature $\left(T_{\mathrm{m}}\right)$, resulting in the fusion of fibers at their points of intersection. Studies have shown that the surface roughness and hydrophobicity are reduced (contact angle reduction of 10\%) following heat treatment. Hot pressing not only reduces the thickness, but also the pore size, pore-size distribution, pore volume, and porosity $(20-30 \%$ reduction), while the fiber diameter is increased (by about $39 \%$ ) due to fiber swelling and morphology smoothing; however improved flux as well as mechanical properties are also observed. The fused fibers are more tightly packed, thereby preventing the penetration of water into the membrane pores, which avoids a reduction in mass-transfer across the membrane and increases the flux. ${ }^{21,40,70,95}$ 
a)

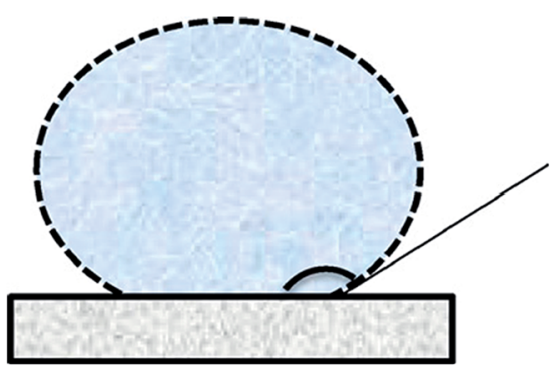

b)

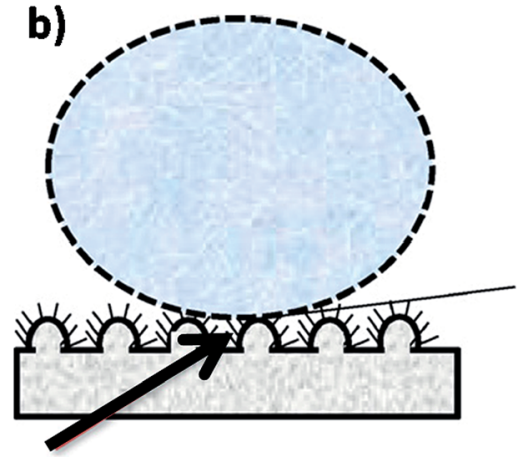

Air pockets

Fig. 15 Examples of water contact angle on (a) a hydrophilic membrane surface and (b) a hydrophobic surface (e.g., after surface modification). Reproduced with permission. Reproduced with permission from ref. 86. Copyright 2014, Elsevier Science Ltd.

Temperature. MD flux increases with increasing flowrate and temperature difference, as shown in Fig. 16.

4.4.4 Modifying ENMs for MD. Although the features of electrospun membranes are more suitable for MD than those fabricated by alternative methods, including their higher hydrophobicities (with increased nanofiber-surface roughness), porosities, and surface areas, they do, however, require further modification for enhanced or improved performance. For example, membrane hydrophobicity is enhanced by surface modification by increasing roughness and through surface chemistry to obtain superhydrophobic properties. ${ }^{\mathbf{1 1 8}}$ Surfacemodification techniques include the incorporation of roughening agents such as hierarchically structured AgNPs, hydrophobic nanoparticles such as $\mathrm{SiO}_{2},{ }^{91}$ clay, or $\mathrm{TiO}_{2}$ NPs, and hydrothermal surface-chemistry enhancements that involve activation prior to the incorporation of NPs, and electroless deposition, among others. However, some surface-modification techniques, such as the latter, can bring about shortcomings, including reduced porosity (but with high permeability) and increased fiber

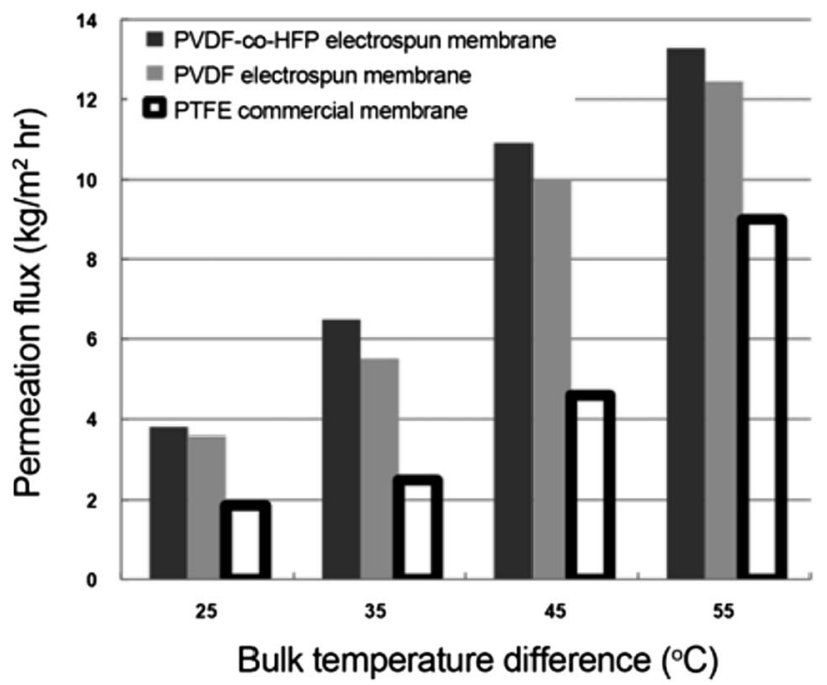

Fig. 16 Plot depicting the effect of temperature difference on MD performance. Reproduced with permission from ref. 69. Copyright 2012, Springer Verlag. diameters. The resulting dense layer of coated NPs tends to create high mass-transfer resistance due to reduced pore sizes, which lowers membrane flux. The incorporation of nanoparticles has been shown to not only enhance hydrophobicity and LEP, but also the thermal and mechanical properties of the membrane. ${ }^{21} \mathrm{TiO}_{2}$ is well dispersed within the polymer matrix and, unlike CNTs that increase pore size, $\mathrm{TiO}_{2}$ reduces pore size.

\subsection{New RO and MD technologies}

One of the latest trends in RO and MD desalination involves the inclusion of nano-sized materials that aid the desalination process. Non-polymeric materials include carbon-based particles (carbon nanotubes (CNTs) and graphene), zeolites, aquaporin (AQP),,$^{\mathbf{8 7 1 1 9 - 1 2 2}}$ and molecular sieves. ${ }^{\mathbf{9 0 , 1 2 3 - 1 2 7}}$ Nanomaterials, such as CNTs, increase nanofiber roughness through the creation of air pockets; ${ }^{\mathbf{1 2 8}}$ they enhance the fluxes of RO TFNC membranes and MD, which increases with higher nanoparticle content. Incorporation of CNTs within the nanofiber polymer matrix provides the added advantage of fiber thinning during electrospinning due to their high conductivities. ${ }^{129}$ However, irrespective of the uniform pore-size distribution (that minimizes wetting), pore size increases with higher CNT content $(\sim 3 \mathrm{wt} \%)$, as does the flux. At this content, irrespective of the higher flux, the large pore sizes result in reduced LEP, which may be due to CNT-nanoparticle agglomeration. Kyoungjin An et al. and $\mathrm{Lee}^{87}$ determined the optimum CNT content to be $0.5 \% \mathrm{wt} \%$, above which agglomeration begins. The unique water-transport properties of CNTs and graphene create nano-channels that facilitate water flow and, as a consequence, result in high-flux membranes, with fluxes that increase with CNT content. ${ }^{87}$ Highly porous zeolites offer cavities for water transport, while the sizes of the pores produced make them exceptional for retaining salt ions from the water stream. In addition to adsorption and sieving, the ion-exchange properties are an added advantage. AQPs are types of biological membrane that also offer effective water transport pathways while retaining ions. These novel materials are incorporated into the polymer matrix of the membrane during fabrication, thereby improving membrane properties, such as permeability 
(hydrophilicity), fouling, and salt permselectivity. For example, graphene-incorporate ENMs exhibit enhanced membrane superhydrophobicity, porosity, and LEP, for MD applications. ${ }^{\mathbf{1 , 8 , 1 3 0 , 1 3 1}}$

\section{Conclusion and future outlook}

Among membrane technologies for water treatment, RO and MD are the most relevant for the separation of salt and are therefore the most commonly used for seawater desalination. While thermally based techniques are first-generation, membrane-based methods are second-generation technologies. RO is the best of the pressure-driven membrane processes, which uses membranes with pore sizes well below $1 \mathrm{~nm}$ that can be formed through surface modification by thin-film coating; hence RO is capable of removing all types of ions, including those of monovalent salts, and is therefore the most suited for desalination applications. MD on the other hand is also theoretically capable of completely removing salts/ $\mathrm{NaCl}(100 \%$ rejection). Nanotechnology involving the use of nanofibers is a fast-growing area of research. As a consequence, electrospinning is considered to be a feasible method for the production of nanomaterial substrates with high surface porosities and interconnected pore structures for pressuredriven and thermally based membrane processes. ENMs have been applied to desalination in limited ways due to their large pores that are incapable of removing salts, which is the reason why these membranes have not been used as top active layers or single-layer self-supported membranes in RO desalination. Electrospun nanofibrous membranes that have undergone crosslinking can directly take part in the RO separation process (i.e., as a top layer). So far, ENMs, as top active selective/barrier layers, have only been successfully used in UF-separation applications, which suggest that future ENM-growth opportunities exist in both MD and RO operating environments.

\section{Conflicts of interest}

There are no conflicts to declare.

\section{Acknowledgements}

The authors would like to thank the Department of Science and Technology (HGERA8X) and the Council for Scientific and Industrial Research (HGER74P), South Africa for financial support.

\section{References}

1 Y. H. Teow and A. W. Mohammad, Desalination, 2017, 356, 226-254.

2 M. A. Shannon, P. W. Bohn, M. Elimelech, J. G. Georgiadis, B. J. Mariñas and A. M. Mayes, Nature, 2008, 452, 301-310.

3 C. Feng, K. C. Khulbe, T. Matsuura, R. Gopal, S. Kaur, S. Ramakrishna and M. Khayet, J. Membr. Sci., 2008, 311, 1-6.

4 M. Elimelech and W. A. Phillip, Science, 2011, 333, 712-717.
5 M. Essalhi and M. Khayet, J. Membr. Sci., 2013, 433, 167179.

6 M. Khayet, M. C. García-Payo, L. García-Fernández and J. Contreras-Martínez, Desalination, 2018, 426, 174-184.

7 K. P. Lee, T. C. Arnot and D. Mattia, J. Membr. Sci., 2011, 370, 1-22.

8 S. Subramanian and R. Seeram, Desalination, 2013, 308, 198-208.

9 S. S. Ray, S.-S. Chen, D. Sangeetha, H.-M. Chang, C. N. D. Thanh, Q. H. Le and H.-M. Ku, Environ. Chem. Lett., 2018, 16, 1-4.

10 Y. Liao, C.-H. Loh, M. Tian, R. Wang and A. G. Fane, Prog. Polym. Sci., 2018, 77, 69-94.

11 S. S. Shenvi, A. M. Isloor and A. F. Ismail, Desalination, 2015, 368, 10-26.

12 R. Gopal, S. Kaur, Z. Ma, C. Chan, S. Ramakrishna and T. Matsuura, J. Membr. Sci., 2006, 281, 581-586.

13 K. Yoon, B. S. Hsiao and B. Chu, J. Mater. Chem., 2008, 18, 5326-5334.

14 A. G. Fane, W. Rong and M. X. Hu, Angew. Chem., Int. Ed., 2015, 54, 3368-3386.

15 K. Yoon, K. Kim, X. Wang, D. Fang, B. S. Hsiao and B. Chu, Polymer, 2006, 47, 2434-2441.

16 Z. Zhao, J. Zheng, M. Wang, H. Zhang and C. C. Han, J. Membr. Sci., 2012, 394-395, 209-217.

17 K. Yoon, B. S. Hsiao and B. Chu, J. Membr. Sci., 2009, 338, 145-152.

18 G. Xuefei, X. Li-Ping, X. Zhongxin, F. Lin, P. Jitao, W. Yongqiang, W. Shutao and Z. Xueji, Adv. Mater., 2014, 26, 1771-1775.

19 Z. Karim, A. P. Mathew, M. Grahn, J. Mouzon and K. Oksman, Carbohydr. Polym., 2014, 112, 668-678.

20 Y. C. Woo, L. D. Tijing, M. J. Park, M. Yao, J.-S. Choi, S. Lee, S.-H. Kim, K.-J. An and H. K. Shon, Desalination, 2017, 403, 187-198.

21 L. D. Tijing, J.-S. Choi, S. Lee, S.-H. Kim and H. K. Shon, J. Membr. Sci., 2014, 453, 435-462.

22 E. Drioli, A. Ali and F. Macedonio, Desalination, 2015, 356, 56-84.

23 M. S. El-Bourawi, Z. Ding, R. Ma and M. Khayet, J. Membr. Sci., 2006, 285, 4-29.

24 H. Maab, L. Francis, A. Al-saadi, C. Aubry, N. Ghaffour, G. Amy and S. P. Nunes, J. Membr. Sci., 2012, 423-424, 1119.

25 B. S. Lalia, V. Kochkodan, R. Hashaikeh and N. Hilal, Desalination, 2013, 326, 77-95.

26 T. Subbiah, G. S. Bhat, R. W. Tock, S. Parameswaran and S. S. Ramkumar, J. Appl. Polym. Sci., 2005, 96, 557-569.

27 F. E. Ahmed, B. S. Lalia and R. Hashaikeh, Desalination, 2015, 356, 15-24.

28 N. Bhardwaj and S. C. Kundu, Biotechnol. Adv., 2010, 28, 325-347.

29 R. S. Barhate, C. K. Loong and S. Ramakrishna, J. Membr. Sci., 2006, 283, 209.

30 A. Haider, S. Haider and I.-K. Kang, Arabian J. Chem., 2015, DOI: 10.1016/j.arabjc.2015.11.015. 
31 S. Huan, G. Liu, G. Han, W. Cheng, Z. Fu, Q. Wu and Q. Wang, Materials, 2015, 8, 2718-2734.

32 H.-J. Choi, S. B. Kim, S. H. Kim and M.-H. Lee, J. Air Waste Manage. Assoc., 2014, 64, 322-329.

33 J. M. Deitzel, J. Kleinmeyer, D. Harris and N. C. Beck Tan, Polymer, 2001, 42, 261-272.

34 C. J. Angammana and S. H. Jayaram, Part. Sci. Technol., 2016, 34, 72-82.

35 D.-G. Yu, J. Zhou, N. P. Chatterton, Y. Li, J. Huang and X. Wang, Int. J. Nanomed., 2012, 7, 5725-5732.

36 Q. P. Pham, U. Sharma and A. G. Mikos, Biomacromolecules, 2006, 7, 2796-2805.

37 K. H. Lee, H. Y. Kim, M. S. Khil, Y. M. Ra and D. R. Lee, Polymer, 2003, 44, 1287-1294.

38 S. S. Ray, S.-S. Chen, C.-W. Li, N. C. Nguyen and H. T. Nguyen, RSC Adv., 2016, 6, 85495-85514.

39 X. Wang, X. Chen, K. Yoon, D. Fang, B. S. Hsiao and B. Chu, Environ. Sci. Technol., 2005, 39, 7684-7691.

40 Y. C. Woo, L. D. Tijing, M. J. Park, M. Yao, J. S. Choi, S. Lee, S. H. Kim, K. J. An and H. K. Shon, Desalination, 2017, 403, 187-198.

41 H. You, Y. Yang, X. Li, K. Zhang, X. Wang, M. Zhu and B. S. Hsiao, J. Membr. Sci., 2012, 394-395, 241-247.

42 X. Wang, H. Ma, B. Chu and B. S. Hsiao, Desalination, 2017, 420, 91-98.

43 G.-R. Xu, J.-N. Wang and C.-J. Li, Desalination, 2013, 328, 83-100.

44 D. Li, Y. Yan and H. Wang, Prog. Polym. Sci., 2016, 61, 104155.

45 W. J. Lau, A. F. Ismail, N. Misdan and M. A. Kassim, Desalination, 2012, 287, 190-199.

46 L. Eykens, I. Hitsov, K. De Sitter, C. Dotremont, L. Pinoy, I. Nopens and B. Van der Bruggen, J. Membr. Sci., 2016, 498, 353-364.

47 M. Elma, C. Yacou, D. K. Wang, S. Smart and J. C. Diniz da Costa, Water, 2012, 4, 629-649.

48 M. M. A. Shirazi, A. Kargari, S. Ramakrishna, J. Doyle, M. Rajendrian and R. Babu, J. Membr. Sci. Res., 2017, 3, 209-227.

49 P. S. Suja, C. R. Reshmi, P. Sagitha and A. Sujith, Polym. Rev., 2017, 57, 467-473.

50 K. Satinderpal, S. Subramanian, G. Renuga and R. Seeram, J. Appl. Polym. Sci., 2012, 124, E205-E215.

51 X. Wang, K. Zhang, Y. Yang, L. Wang, Z. Zhou, M. Zhu, B. S. Hsiao and B. Chu, J. Membr. Sci., 2010, 356, 110-116.

52 M. I. Litter, W. Choi, D. D. Dionysiou, P. Falaras, A. Hiskia, G. Li Puma, T. Pradeep and J. Zhao, J. Hazard. Mater., 2012, 211-212, 1-2.

53 X. Qu, P. J. J. Alvarez and Q. Li, Water Res., 2013, 47, 39313946.

54 M. Wiesner, Q. Li, J. Burgess, R. Kaegi and D. Dixon, Water Res., 2013, 47, 3865-4206.

55 Y. C. Woo, L. D. Tijing, W.-G. Shim, J.-S. Choi, S.-H. Kim, T. He, E. Drioli and H. K. Shon, J. Membr. Sci., 2016, 520, 99-110.

56 C. Feng, K. C. Khulbe, T. Matsuura, S. Tabe and A. F. Ismail, Sep. Purif. Technol., 2013, 102, 118-128.
57 M. Mavroudi, S. P. Kaldis and G. P. Sakellaropoulos, J. Membr. Sci., 2006, 272, 103-115.

58 M. Essalhi and M. Khayet, J. Membr. Sci., 2013, 433, 180191.

59 C. Burger, B. S. Hsiao and B. Chu, Annu. Rev. Mater. Res., 2006, 36, 333-368.

60 L. A. Hoover, J. D. Schiffman and M. Elimelech, Desalination, 2013, 308, 73-81.

61 A. Amarjargal, L. D. Tijing, M. T. G. Ruelo, D. H. Lee and C. S. Kim, Mater. Chem. Phys., 2012, 135, 277-281.

62 L. D. Tijing, C.-H. Park, S.-J. Kang, A. Amarjargal, T.-H. Kim, H. R. Pant, H. J. Kim, D. H. Lee and C. S. Kim, Appl. Surf. Sci., 2013, 264, 453-457.

63 K. Yu Wang, T.-S. Chung and M. Gryta, Chem. Eng. Sci., 2008, 63, 2587-2594.

64 J. Phattaranawik, R. Jiraratananon and A. G. Fane, J. Membr. Sci., 2003, 215, 75.

65 A. S. Kim, J. Membr. Sci., 2013, 428, 410-424.

66 A. T. Servi, J. Kharraz, D. Klee, K. Notarangelo, B. Eyob, E. Guillen-Burrieza, A. Liu, H. A. Arafat and K. K. Gleason, J. Membr. Sci., 2016, 520, 850-859.

67 A. Singh, L. Steely and H. R. Allcock, Langmuir, 2005, 21, 11604-11607.

68 A. Alkhudhiri, N. Darwish and N. Hilal, Desalination, 2012, 287, 2-18.

69 C.-I. Su, J.-H. Shih, M.-S. Huang, C.-M. Wang, W.-C. Shih and Y.-s. Liu, Fibers Polym., 2012, 13, 698-702.

70 B. S. Lalia, E. Guillen-Burrieza, H. A. Arafat and R. Hashaikeh, J. Membr. Sci., 2013, 428, 104-115.

71 M. Ma, R. M. Hill, J. L. Lowery, S. V. Fridrikh and G. C. Rutledge, Langmuir, 2005, 21, 5549-5554.

72 A. Kazim, S. Eren, O. Y. Cleva and Y. Z. Menceloglu, Angew. Chem., Int. Ed., 2004, 43, 5210-5213.

73 A. E. Deniz, H. A. Vural, B. Ortaç and T. Uyar, Mater. Lett., 2011, 65, 2941-2943.

74 A. Sonseca, L. Peponi, O. Sahuquillo, J. M. Kenny and E. Giménez, Polym. Degrad. Stab., 2012, 97, 2052-2059.

75 S. Wang, C. Wang, B. Zhang, Z. Sun, Z. Li, X. Jiang and X. Bai, Mater. Lett., 2010, 64, 9-13.

76 D. Wu, T. Shi, T. Yang, Y. Sun, L. Zhai, W. Zhou, M. Zhang and J. Zhang, Eur. Polym. J., 2011, 47, 284-293.

77 L. D. Tijing, A. Amarjargal, Z. Jiang, M. T. G. Ruelo, C.-H. Park, H. R. Pant, D.-W. Kim, D. H. Lee and C. S. Kim, Curr. Appl. Phys., 2013, 13, 205-210.

78 A. Amarjargal, L. D. Tijing, I.-T. Im and C. S. Kim, Chem. Eng. J., 2013, 226, 243-254.

79 M. Safarpour, V. Vatanpour, A. Khataee, H. Zarrabi, P. Gholami and M. E. Yekavalangi, Desalination, 2017, 411, 89-100.

80 A. Garofalo, M. C. Carnevale, L. Donato, E. Drioli, O. Alharbi, S. A. Aljlil, A. Criscuoli and C. Algieri, Desalination, 2016, 397, 205-212.

81 A. Garofalo, L. Donato, E. Drioli, A. Criscuoli, M. C. Carnevale, O. Alharbi, S. A. Aljlil and C. Algieri, Sep. Purif. Technol., 2014, 137, 28-35.

82 Y. Zhu, K. M. Gupta, Q. Liu, J. Jiang, J. Caro and A. Huang, Desalination, 2016, 385, 75-82. 
83 S. M. Hosseini, S. Rafiei, A. R. Hamidi, A. R. Moghadassi and S. S. Madaeni, Desalination, 2014, 351, 138-144.

84 P. Swenson, B. Tanchuk, E. Bastida, W. An and S. M. Kuznicki, Desalination, 2012, 286, 442-446.

85 L.-F. Ren, F. Xia, J. Shao, X. Zhang and J. Li, Desalination, 2017, 404, 155-166.

86 Y. Liao, R. Wang and A. G. Fane, J. Membr. Sci., 2013, 440, 77-87.

87 A. Kyoungjin An, E.-J. Lee, J. Guo, S. Jeong, J.-G. Lee and N. Ghaffour, Sci. Rep., 2017, 7, 41562.

88 E.-J. Lee, A. K. An, T. He, Y. C. Woo and H. K. Shon, J. Membr. Sci., 2016, 520, 145.

89 L. Francis, H. Maab, A. AlSaadi, S. Nunes, N. Ghaffour and G. L. Amy, Desalin. Water Treat., 2013, 51, 1337-1343.

90 Q. Lyu, S. Sun, C. Li, S. Hu and L.-C. Lin, ACS Appl. Mater. Interfaces, 2018, 10, 18778-18785.

91 Y. Liao, R. Wang and A. G. Fane, Environ. Sci. Technol., 2014, 48, 6335-6341.

92 X. Li, C. Wang, Y. Yang, X. Wang, M. Zhu and B. S. Hsiao, ACS Appl. Mater. Interfaces, 2014, 6, 2423-2430.

93 J. Lee, C. Boo, W.-H. Ryu, A. D. Taylor and M. Elimelech, ACS Appl. Mater. Interfaces, 2016, 8, 11154-11161.

94 M. Yao, Y. C. Woo, L. D. Tijing, W. G. Shim, J. S. Choi, S. H. Kim and H. K. Shon, Desalination, 2016, 378, 80-91.

95 Y. Liao, R. Wang, M. Tian, C. Qiu and A. G. Fane, J. Membr. Sci., 2013, 425-426, 30-39.

96 J. A. Prince, G. Singh, D. Rana, T. Matsuura, V. Anbharasi and T. S. Shanmugasundaram, J. Membr. Sci., 2012, 397398, 80-86.

97 A. Razmjou, E. Arifin, G. Dong, J. Mansouri and V. Chen, J. Membr. Sci., 2012, 415-416, 850-863.

98 D. Han and A. J. Steckl, Langmuir, 2009, 25, 9454-9462.

99 S. S. Ray, S. S. Chen, N. C. Nguyen, H. T. Hsu, H. T. Nguyen and C. T. Chang, Desalination, 2017, 414, 18-27.

100 H. Fang, J. F. Gao, H. T. Wang and C. S. Chen, J. Membr. Sci., 2012, 403-404, 41-46.

101 F. A. Banat and J. Simandl, Desalination, 1994, 95, 39-52.

102 N.-N. Bui, M. L. Lind, E. M. V. Hoek and J. R. McCutcheon, J. Membr. Sci., 2011, 385-386, 10-19.

103 M. Su, M. M. Teoh, K. Y. Wang, J. Su and T.-S. Chung, J. Membr. Sci., 2010, 364, 278-289.

104 N. Tang, Q. Jia, H. Zhang, J. Li and S. Cao, Desalination, 2010, 256, 27-36.

105 M. Yao, Y. C. Woo, L. D. Tijing, W.-G. Shim, J.-S. Choi, S.-H. Kim and H. K. Shon, Desalination, 2016, 378, 80-91.

106 E.-J. Lee, A. K. An, P. Hadi, S. Lee, Y. C. Woo and H. K. Shon, J. Membr. Sci., 2017, 524, 712-720.

107 M. Obaid, Z. K. Ghouri, O. A. Fadali, K. A. Khalil, A. A. Almajid and N. A. M. Barakat, ACS Appl. Mater. Interfaces, 2016, 8, 4561-4574.
108 S. M. Seyed Shahabadi, H. Rabiee, S. M. Seyedi, A. Mokhtare and J. A. Brant, J. Membr. Sci., 2017, 537, 140-150.

109 M. Essalhi and M. Khayet, J. Membr. Sci., 2012, 417-418, 163-176.

110 L. D. Tijing, Y. C. Woo, J.-S. Choi, S. Lee, S.-H. Kim and H. K. Shon, J. Membr. Sci., 2015, 475, 215-244.

111 A. K. An, J. Guo, S. Jeong, E.-J. Lee, S. A. A. Tabatabai and T. Leiknes, Water Res., 2016, 103, 362-371.

112 F. Shi, Z. Wang and X. Zhang, Adv. Mater., 2005, 17, 10051009.

113 M. Ma and R. M. Hill, Curr. Opin. Colloid Interface Sci., 2006, 11, 193-202.

114 K. Teshima, H. Sugimura, Y. Inoue, O. Takai and A. Takano, Appl. Surf. Sci., 2005, 244, 619-622.

115 B. Bhushan and Y. Chae Jung, Ultramicroscopy, 2007, 107, 1033-1041.

116 M. Khayet, K. C. Khulbe and T. Matsuura, J. Membr. Sci., 2004, 238, 199-211.

117 R. W. Schofield, A. G. Fane and C. J. D. Fell, J. Membr. Sci., 1990, 53, 159-171.

118 N. J. Shirtcliffe, G. McHale, S. Atherton and M. I. Newton, Adv. Colloid Interface Sci., 2010, 161, 124-138.

119 Z. Yang, X.-H. Ma and C. Y. Tang, Desalination, 2018, 434, 37-59.

120 M. Kumar, M. Grzelakowski, J. Zilles, M. Clark and W. Meier, Proc. Natl. Acad. Sci. U. S. A., 2007, 104, 2071920724.

121 R. Das, M. E. Ali, S. B. A. Hamid, S. Ramakrishna and Z. Z. Chowdhury, Desalination, 2014, 336, 97-109.

122 M. L. Lind, A. K. Ghosh, A. Jawor, X. Huang, W. Hou, Y. Yang and E. M. V. Hoek, Langmuir, 2009, 25, 1013910145.

123 J. Schrier, ACS Appl. Mater. Interfaces, 2012, 4, 3745-3752.

124 D. Cohen-Tanugi, L.-C. Lin and J. C. Grossman, Nano Lett., 2016, 16, 1027-1033.

125 K. Sint, B. Wang and P. Král, J. Am. Chem. Soc., 2008, 130, 16448-16449.

126 S. C. O'Hern, M. S. H. Boutilier, J.-C. Idrobo, Y. Song, J. Kong, T. Laoui, M. Atieh and R. Karnik, Nano Lett., 2014, 14, 1234-1241.

127 D. Cohen-Tanugi and J. C. Grossman, Nano Lett., 2012, 12, 3602-3608.

128 J. A. Prince, V. Anbharasi, T. S. Shanmugasundaram and G. Singh, Sep. Purif. Technol., 2013, 118, 598-603.

129 Z. H. Liu, C. T. Pan, L. W. Lin and H. W. Lai, Sens. Actuators, A, 2013, 193, 13-24.

130 E. Wibowo, Sutisna, M. Rokhmat, R. Murniati, Khairurrijal and M. Abdullah, Procedia Eng., 2017, 170, 8-13.

131 C. H. Cho, K. Y. Oh, S. K. Kim, J. G. Yeo and P. Sharma, J. Membr. Sci., 2011, 371, 226-238. 